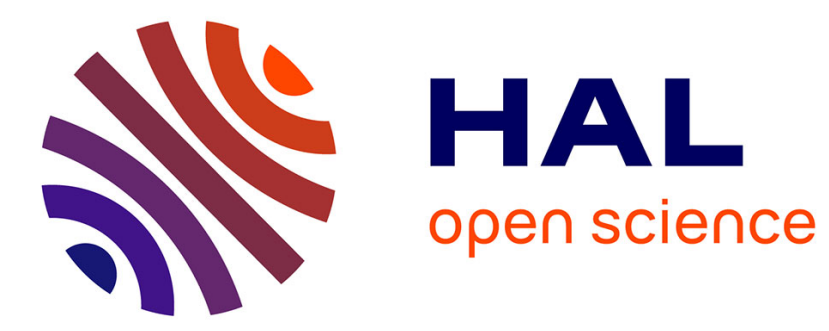

\title{
Dynamic Programming and Error Estimates for Stochastic Control Problems with Maximum Cost
}

Olivier Bokanowski, Athena Picarelli, Hasnaa Zidani

\section{To cite this version:}

Olivier Bokanowski, Athena Picarelli, Hasnaa Zidani. Dynamic Programming and Error Estimates for Stochastic Control Problems with Maximum Cost. Applied Mathematics and Optimization, 2015, 71 (1), pp.125-163. 10.1007/s00245-014-9255-3 . hal-00931025v2

\section{HAL Id: hal-00931025 \\ https://hal.inria.fr/hal-00931025v2}

Submitted on 11 May 2014

HAL is a multi-disciplinary open access archive for the deposit and dissemination of scientific research documents, whether they are published or not. The documents may come from teaching and research institutions in France or abroad, or from public or private research centers.
L'archive ouverte pluridisciplinaire HAL, est destinée au dépôt et à la diffusion de documents scientifiques de niveau recherche, publiés ou non, émanant des établissements d'enseignement et de recherche français ou étrangers, des laboratoires publics ou privés. 


\title{
DYNAMIC PROGRAMMING AND ERROR ESTIMATES FOR STOCHASTIC CONTROL PROBLEMS WITH MAXIMUM COST
}

\author{
OLIVIER BOKANOWSKI, ATHENA PICARELLI, AND HASNAA ZIDANI
}

\begin{abstract}
This work is concerned with stochastic optimal control for a running maximum cost. A direct approach based on dynamic programming techniques is studied leading to the characterization of the value function as the unique viscosity solution of a second order HamiltonJacobi-Bellman (HJB) equation with an oblique derivative boundary condition. A general numerical scheme is proposed and a convergence result is provided. Error estimates are obtained for the semi-Lagrangian scheme. These results can apply to the case of lookback options in finance. Moreover, optimal control problems with maximum cost arise in the characterization of the reachable sets for a system of controlled stochastic differential equations. Some numerical simulations on examples of reachable analysis are included to illustrate our approach.
\end{abstract}

AMS subject classifications: 49J20, 49L25, 65M15, 35K55

Keywords: Hamilton-Jacobi equations, oblique Neuman boundary condition, error estimate, viscosity notion, reachable sets under state constraints, lookback options, maximum cost.

\section{INTRODUCTION}

Consider the controlled process $X_{t, x}^{u}(\cdot)$ solution of the stochastic differential equation

$$
\left\{\begin{array}{l}
d X(s)=b(s, X(s), u(s)) d s+\sigma(s, X(s), u(s)) d \mathcal{B}(s) \quad s \in[t, T] \\
X(t)=x
\end{array}\right.
$$

(where $u \in \mathcal{U}$ denotes the control process). We are interested in an optimal control problem where the cost function is given by :

$$
J(t, x, u)=\mathbb{E}\left[\psi\left(X_{t, x}^{u}(T), \max _{s \in[t, T]} g\left(X_{t, x}^{u}(s)\right)\right)\right],
$$

and the value function is defined by:

$$
v(t, x):=\inf _{u \in \mathcal{U}} J(t, x, u) .
$$

Date: May 1, 2014.

This work was partially supported by the EU under the 7th Framework Programme Marie Curie Initial Training Network "FP7-PEOPLE-2010-ITN", SADCO project, GA number 264735-SADCO. 
This problem arises from the study of some path-dependent options in finance (lookback options). Another application of control problem with maximum running cost comes from the characterization of reachable sets for state-constrained controlled stochastic systems (see section 2.3).

The main contributions to the study of this kind of problems can be found in [4] and [11] (see also [26] for the stationary case in the framework of classical solutions). In these works the dynamic programming techniques are applied on the $L^{p}$-approximation of the $L^{\infty}$-cost functional $(1.2)$, using the approximation:

$$
a \vee b \simeq\left(a^{p}+b^{p}\right)^{\frac{1}{p}} \quad(\text { for } p \rightarrow \infty)
$$

for any $a, b \geq 0$, where $a \vee b:=\max (a, b)$. Then a characterization of the auxiliary value function $v$ (see definition (2.4) ) as solution to a suitable Hamilton-Jacobi equation is obtained as limit for $p \rightarrow \infty$. A fundamental hypothesis in order to apply this approach is the positivity of the functions involved.

In our work, a direct derivation of a Dynamic Programming Principle for the function $v$ gives an alternative and natural way for dealing with the maximum running cost problems under less restrictive assumptions. By this way, the optimal control problem $(1.3)$ is connected to the solution of a HJB equation with oblique derivative boundary conditions. Here, the boundary conditions have to be understood in the viscosity sense (see [27]).

The second part of the paper is devoted to numerical aspects. First, a general scheme is proposed to deal with HJB equations with oblique derivative boundary conditions. Then, a convergence result is proved by using the general framework of Barles-Souganidis [10] based on the monotonicity, stability, consistency of the scheme (a precise definition of these notions, in the case of HJB equations with oblique derivative boundary condition, is given in section 4.1). Secondly, we focus on the fully discrete semi-Lagrangian scheme and we study some properties of the numerical approximation.

Semi-Lagrangian schemes for second order HJB equations (in the general form of Markov chain approximations) have been first considered in the book [32]. In the framework of viscosity solutions, semi-Lagrangian schemes for first order HJB equations have been studied in [20]. Extensions to the second order case can be found in [35, 19, 23, 24]. In our context, the numerical scheme that will be studied couples the classical semi-Lagrangian scheme with an additional projection step on the boundary. For this particular scheme, we derive a rate of convergence, generalizing in this way the results already known for general HJB equations without boundary conditions. More precisely, an error bound of the order of $h^{1 / 4}$ (where $h$ is the time step) is obtained for Lipschitz (unbounded) cost function. The activity related to error estimate for numerical approximations of nonlinear second order degenerate equations dated back few years ago with [30, 31] where some useful techniques of "shaking coefficients" were introduced, and then 
mollifiers were used to get smooth sub-solutions. These ideas were later used extensively by many authors [6, 7, 8, 14].

The paper is organized as follows: Section 2 introduces the problem and some motivations. In particular it will be shown how it is possible to connect a state-constrained reachability problem to an optimal control problem associated to a cost functional of the form 1.2 . Section 3 is devoted to the characterization of the value function by the appropriate HJB equation. A strong comparison principle is also stated. In Section 4 the numerical approximation is discussed and a general convergence result is provided. The semi-Lagrangian scheme is presented in Section 4.2 and the properties of this scheme are investigated. Section 5 focuses on the study of error estimates. Finally some numerical tests are presented in Section 6 to analyse the relevance of the proposed scheme.

\section{Formulation of the Problem}

2.1. Setting and preliminary results. Let $(\Omega, \mathcal{F}, \mathbb{P})$ be a probability space, $\left\{\mathcal{F}_{t}, t \geq 0\right\}$ a filtration on $\mathcal{F}$ and $\mathcal{B}(\cdot)$ a Brownian motion in $\mathbb{R}^{p}$ (with $p \geq 1)$.

Given $T>0$ and $0 \leq t \leq T$, the following system of controlled stochastic differential equations (SDEs) in $\mathbb{R}^{d}(d \geq 1)$ is considered

$$
\left\{\begin{array}{l}
d X(s)=b(s, X(s), u(s)) d s+\sigma(s, X(s), u(s)) d \mathcal{B}(s) \quad s \in[t, T] \\
X(t)=x,
\end{array}\right.
$$

where $u \in \mathcal{U}$ set of progressively measurable processes with values in $U \subset$ $\mathbb{R}^{m}(m \geq 1), U$ is assumed to be a compact set. The following classical assumption will be considered on the coefficients $b$ and $\sigma$ :

(H1) $b:[0, T] \times \mathbb{R}^{d} \times U \rightarrow \mathbb{R}^{d}$ and $\sigma:[0, T] \times \mathbb{R}^{d} \times U \rightarrow \mathbb{R}^{d \times p}$ are continuous functions. Moreover, there exist $L_{b}, L_{\sigma}>0$ such that for every $x, y \in \mathbb{R}^{d}, t \in[0, T], u \in U$ :

$$
|b(t, x, u)-b(t, y, u)| \leq L_{b}|x-y|, \quad|\sigma(t, x, u)-\sigma(t, y, u)| \leq L_{\sigma}|x-y| .
$$

We recall some classical results on (2.1) (see [39]):

Theorem 2.1. Under assumption (H1) there exists a unique $\mathcal{F}_{t}$-adapted process, denoted by $X_{t, x}^{u}(\cdot)$, strong solution of (2.1). Moreover there exists a constant $C_{0}>0$, such that for any $u \in \mathcal{U}, 0 \leq t \leq t^{\prime} \leq T$ and $x, x^{\prime} \in \mathbb{R}^{d}$

$$
\begin{aligned}
\mathbb{E}\left[\sup _{\theta \in\left[t, t^{\prime}\right]}\left|X_{t, x}^{u}(\theta)-X_{t, x^{\prime}}^{u}(\theta)\right|^{2}\right] & \leq C_{0}^{2}\left|x-x^{\prime}\right|^{2}, \\
\mathbb{E}\left[\sup _{\theta \in\left[t, t^{\prime}\right]}\left|X_{t, x}^{u}(\theta)-X_{t^{\prime}, x}^{u}(\theta)\right|^{2}\right] & \leq C_{0}^{2}\left(1+|x|^{2}\right)\left|t-t^{\prime}\right|, \\
\mathbb{E}\left[\sup _{\theta \in\left[t, t^{\prime}\right]}\left|X_{t, x}^{u}(\theta)-x\right|^{2}\right] & \leq C_{0}^{2}\left(1+|x|^{2}\right)\left|t-t^{\prime}\right| .
\end{aligned}
$$


Consider two functions $\psi, g$ such that:

(H2) $\psi: \mathbb{R}^{d} \times \mathbb{R} \rightarrow \mathbb{R}$ and $g: \mathbb{R}^{d} \rightarrow \mathbb{R}$ are Lipschitz functions. Their Lipschitz constants will be denoted by $L_{\psi}$ and $L_{g}$ respectively.

In this paper we deal with optimal control problems of the form

$$
v(t, x):=\inf _{u \in \mathcal{U}} \mathbb{E}\left[\psi\left(X_{t, x}^{u}(T), \max _{s \in[t, T]} g\left(X_{t, x}^{u}(s)\right)\right)\right] .
$$

Under assumptions (H1) and (H2), $v$ is a Lipschitz continuous function in $x$ and a $\frac{1}{2}$-Hölder continuous function in $t$ (by the same arguments as in Proposition 2.2 below). In order to characterize the function $v$ as solution of an HJB equation the main tool is the well-known optimality principle. The particular non-Markovian structure of the cost functional in 2.3 prohibits the direct use of the standard techniques. To avoid this difficulty it is classical to reformulate the problem by adding a new variable $y \in \mathbb{R}$ that, roughly speaking, keeps the information of the running maximum. For this reason, we introduce an auxiliary value function $\vartheta$ defined on $[0, T] \times \mathbb{R}^{d} \times \mathbb{R}$ by:

$$
\vartheta(t, x, y):=\inf _{u \in \mathcal{U}} \mathbb{E}\left[\psi\left(X_{t, x}^{u}(T), \max _{s \in[t, T]} g\left(X_{t, x}^{u}(s)\right) \vee y\right)\right]
$$

The following property holds:

$$
\vartheta(t, x, g(x))=v(t, x),
$$

so from now on, we will only work with the value function $\vartheta$ since the value of $v$ can be obtained by 2.5 .

Proposition 2.2. Under assumptions (H1) and (H2), $\vartheta$ is a Lipschitz continuous function in $(x, y)$ uniformly with respect to $t$, and a $\frac{1}{2}$-Hölder continuous function in $t$. More precisely, there exists $L_{\vartheta}>0$ ( $L_{\vartheta}$ depends only on $\left.C_{0}, L_{\psi}, L_{g}\right)$ such that:

$$
\left|\vartheta(t, x, y)-\vartheta\left(t^{\prime}, x^{\prime}, y^{\prime}\right)\right| \leq L_{\vartheta}\left(\left|x-x^{\prime}\right|+\left|y-y^{\prime}\right|+(1+|x|)\left|t-t^{\prime}\right|^{\frac{1}{2}}\right),
$$

for all $(x, y),\left(x^{\prime}, y^{\prime}\right) \in \mathbb{R}^{d+1}$ and for any $t \leq t^{\prime} \in[0, T]$.

Proof. Let be $t \leq t^{\prime} \leq T$ and $x, x^{\prime} \in \mathbb{R}^{d}$. Notice that the following property holds for the maximum

$$
|(a \vee b)-(c \vee d)| \leq|a-c| \vee|b-d|
$$

Then, the inequalities $(2.2)$ yield to:

$$
\left|\vartheta(t, x, y)-\vartheta\left(t, x^{\prime}, y\right)\right| \leq K \sup _{u \in \mathcal{U}} \mathbb{E}\left[\sup _{s \in[t, T]}\left|X_{t, x}^{u}(s)-X_{t, x^{\prime}}^{u}(s)\right|\right] \leq K C_{0}\left|x-x^{\prime}\right|
$$


for $K=L_{\psi}\left(L_{g}+1\right)$. In a similar way, we obtain

$$
\begin{aligned}
\left|\vartheta(t, x, y)-\vartheta\left(t^{\prime}, x, y\right)\right| \leq & L_{\psi} \sup _{u \in \mathcal{U}} \mathbb{E}\left[\left|X_{t, x}^{u}(T)-X_{t^{\prime}, x}^{u}(T)\right|+L_{g} \sup _{s \in\left[t, t^{\prime}\right]}\left|X_{t, x}^{u}(s)-x\right|+\right. \\
& \left.+L_{g} \sup _{s \in\left[t^{\prime}, T\right]}\left|X_{t^{\prime}, x}^{u}(s)-X_{t^{\prime}, X_{t, x}^{u}\left(t^{\prime}\right)}^{u}(s)\right|\right] \\
\leq & K^{\prime}(1+|x|)\left|t-t^{\prime}\right|^{\frac{1}{2}}
\end{aligned}
$$

for a positive constant $K^{\prime}>0$ that depends only on $L_{\psi}, L_{g}$ and $C_{0}$. The $L_{\psi}$-Lipschitz behavior in the variable $y$ is immediate. We conclude then the result with $L_{\vartheta}=K \vee K^{\prime} \vee L_{\psi}$.

2.2. Summary of the main results. The first important theoretical result is contained in Theorem 3.2 where it is established that $\vartheta$ is a viscosity solution (in the sense of Definition 3.3) of the following HJB equation with oblique derivative boundary conditions

$$
\begin{cases}-\partial_{t} \vartheta+H\left(t, x, D_{x} \vartheta, D_{x}^{2} \vartheta\right)=0 & \text { in }[0, T) \times D \\ -\partial_{y} \vartheta=0 & \text { on }[0, T) \times \partial D \\ \vartheta(T, x, y)=\psi(x, y) & \text { in } \bar{D}\end{cases}
$$

for a suitable choice of the domain $D \subset \mathbb{R}^{d+1}$.

Theorem 3.4 states a comparison principle for (2.6), adapting the result of [25] to parabolic equations in unbounded domains. Thanks to this result, $\vartheta$ is finally characterized as the unique continuous viscosity solution of the HJB equation.

Starting from Section 4 the numerical aspects will be discussed. A numerical scheme for the approximation of $\vartheta$ will be proposed. Theorem 4.1 gives a general convergence result under hypotheses of stability, consistency and monotonicity.

Error estimates for the semi-discrete semi-Lagrangian scheme are investigated in the Theorems 5.4 and 5.5 , while the rate of convergence for the fully discrete scheme is given in Theorem 5.6.

\subsection{Motivations.}

Reachability for state constrained control systems. A first motivation comes from the reachability analysis for state-constrained stochastic controlled systems. Let $\mathcal{C} \subseteq \mathbb{R}^{d}$ be a target set, and let $\mathcal{K} \subseteq \mathbb{R}^{d}$ be a set of state constraints. Let $d_{\mathcal{C}}$ (resp. $d_{\mathcal{K}}$ ) denotes the Euclidean distance functions from $\mathcal{C}$ (resp. $\mathcal{K}$ ). The aim is to characterize and compute the backward reachable set $\mathcal{R}_{t}^{\mathcal{C}, \mathcal{K}}$ defined by

$$
\begin{aligned}
\mathcal{R}_{t}^{\mathcal{C}, \mathcal{K}}:=\{x \in & \mathbb{R}^{d}, \forall \varepsilon>0, \exists u_{\varepsilon} \in \mathcal{U} \text { such that } \\
& \left.\mathbb{E}\left[d_{\mathcal{C}}\left(X_{t, x}^{u_{\varepsilon}}(T)\right)\right] \leq \varepsilon \text { and } \mathbb{E}\left[\max _{s \in[t, T]} d_{\mathcal{K}}\left(X_{t, x}^{u_{\varepsilon}}(s)\right)\right] \leq \varepsilon\right\} .
\end{aligned}
$$


Remark 2.3. Straightforward calculations yield to the following formulation:

$$
\mathcal{R}_{t}^{\mathcal{C}, \mathcal{K}} \equiv\left\{x \in \mathbb{R}^{d}, \forall \varepsilon>0, \exists u_{\varepsilon} \in \mathcal{U}, \mathbb{E}\left[d_{\mathcal{C}}\left(X_{t, x}^{u_{\varepsilon}}(T)\right) \vee \max _{s \in[t, T]} d_{\mathcal{K}}\left(X_{t, x}^{u_{\varepsilon}}(s)\right)\right] \leq \varepsilon\right\}
$$

Indeed, this is easily deduced from the following inequalities:

$$
\begin{array}{r}
\mathbb{E}\left[d_{\mathcal{C}}\left(X_{t, x}^{u_{\varepsilon}}(T)\right)\right] \vee \mathbb{E}\left[\max _{s \in[t, T]} d_{\mathcal{K}}\left(X_{t, x}^{u_{\varepsilon}}(s)\right)\right] \leq \mathbb{E}\left[d_{\mathcal{C}}\left(X_{t, x}^{u_{\varepsilon}}(T)\right) \vee \max _{s \in[t, T]} d_{\mathcal{K}}\left(X_{t, x}^{u_{\varepsilon}}(s)\right)\right] \\
\leq \mathbb{E}\left[d_{\mathcal{C}}\left(X_{t, x}^{u_{\varepsilon}}(T)\right)\right]+\mathbb{E}\left[\max _{s \in[t, T]} d_{\mathcal{K}}\left(X_{t, x}^{u_{\varepsilon}}(s)\right)\right] .
\end{array}
$$

Many works in the literature have been devoted to the reachability analysis for general stochastic controlled systems, we refer to [2, 3]. Stochastic target problems arising in finance have been also analysed [37, 17]. Here, we suggest to characterize the reachable sets by using the level set approach introduced by Osher and Sethian in [36] in the deterministic case. At the basis of this approach there is the idea of considering the set $\mathcal{R}_{t}^{\mathcal{C}, \mathcal{K}}$ as a level set of a continuous function defined as the value function of a suitable optimal control problem. Recently, the level-set approach has been extended to deal with general deterministic optimal control problems in presence of state constraints, see for example [12, 1]. The level set approach can be also extended to the stochastic setting by considering the following optimal control problem:

$$
\bar{v}(t, x):=\inf _{u \in \mathcal{U}} \mathbb{E}\left[d_{\mathcal{C}}\left(X_{t, x}^{u}(T)\right) \vee \max _{s \in[t, T]} d_{\mathcal{K}}\left(X_{t, x}^{u}(s)\right)\right] .
$$

Then the following equivalence can easily be proved

Theorem 2.4. Under assumption (H1), for every $t \geq 0$, we have:

$$
x \in \mathcal{R}_{t}^{\mathcal{C}, \mathcal{K}} \Longleftrightarrow \bar{v}(t, x)=0 .
$$

Proof. The result follows immediately from Remark 2.3 .

In view of Theorem 2.4 the reachable set $\mathcal{R}_{t}^{\mathcal{C}, \mathcal{K}}$ can be characterized by means of the value function of a control problem with supremum cost in the form of (2.3), with functions $g$ and $\psi$ defined by: $g(x):=d_{\mathcal{K}}(x)$ and $\psi(x, y):=d_{\mathcal{C}}(x) \vee y$.

Lookback options in finance. Another interest for computing expectation of supremum cost functionals is the lookback options in Finance. The value of such an option is typically of the form

$$
\left.\widetilde{v}(t, x)=\mathbb{E}\left[e^{-\int_{t}^{T} r(s) d s} \psi\left(X_{T}, \max _{\theta \in[t, T]} g\left(X_{\theta}\right)\right)\right)\right],
$$

where $X_{\theta}$ (the "asset") is a solution of a one-dimensional SDE (2.1), $g(x)=$ $x, r(\cdot)$ is the interest rate, and $\psi$ is the payoff function. Here the option value depends not only on the value of the asset at time $T$ but also on all 
the values taken between times $t$ and $T$. A detailed description of this model can be found in [38]. Typical payoff functions are $\psi(x, y)=y-x$ (lookback floating strike put), $\psi(x, y)=\max (y-E, 0)$ (fixed strike lookback call), $\psi(x, y)=\max (\min (y, E)-x, 0)$ (lookback limited-risk put), etc., see [38, 5] (see also [4] for other examples and related american lookback options).

\section{The HJB EquATion}

In all the sequel, we will use the abbreviation a.s. for almost surely. Also, almost every will be abbreviated as a.e.. The abbreviation usc (resp. lsc) will be used for upper semi-continuous (resp. lower semi-continuous).

3.1. Dynamic Programming. The aim of this section is to characterize the value function $\vartheta$ defined by (2.4) as the unique viscosity solution of an HJB equation. Let us define the process

$$
Y_{t, x, y}^{u}(\theta):=\max _{s \in[t, \theta]} g\left(X_{t, x}^{u}(s)\right) \vee y,
$$

then the optimal control problem $(2.4)$ can be re-written as

$$
\vartheta(t, x, y):=\inf _{u \in \mathcal{U}} \mathbb{E}\left[\psi\left(X_{t, x}^{u}(T), Y_{t, x, y}^{u}(T)\right)\right] .
$$

Stochastic optimal control problems with running maximum cost in the viscosity solutions framework have been studied in [4, 11. The arguments developed in that papers are based on the approximation technique of the $L^{\infty}$-norm and they only apply if $\psi$ and $g$ are positive functions. Here, we derive the HJB equation directly without using any approximation. For this, the first step is the Bellman's principle that we state in the following theorem.

Theorem 3.1 (Dynamic Programming Principle). Under hypothesis (H1) and (H2), for all $(t, x) \in[0, T) \times \mathbb{R}^{d}$ and all family of stopping times $\left\{\theta^{u}, u \in \mathcal{U}\right\}$ independent of $\mathcal{F}_{t}$ with values on $[t, T]$ :

$$
\vartheta(t, x, y)=\inf _{u \in \mathcal{U}} \mathbb{E}\left[\vartheta\left(\theta^{u}, X_{t, x}^{u}\left(\theta^{u}\right), Y_{t, x, y}^{u}\left(\theta^{u}\right)\right)\right] .
$$

A proof of Theorem 3.1 can be obtained by adapting the same arguments developed by Bouchard and Touzi in [18], thanks to the fact that the couple of variables $\left(X_{t, x}^{u}(\cdot), Y_{t, x, y}^{u}(\cdot)\right)$ satisfies the following fundamental property

$$
\left(\begin{array}{l}
X_{t, x}^{u}(s) \\
Y_{t, x, y}^{u}(s)
\end{array}\right)=\left(\begin{array}{l}
X_{\theta, X_{t, x}^{u}(\theta)}^{u}(s) \\
Y_{\theta, X_{t, x}^{u}(\theta), Y_{t, x, y}^{u}(\theta)}^{u}(s)
\end{array}\right) \quad \text { a.s. }
$$

for any stopping time $\theta$ with $t \leq \theta \leq s \leq T$. In our case the proof is even simpler than the one of [18] thanks to the uniform continuity of $\vartheta$ (Proposition 2.2). 
3.2. Hamilton-Jacobi-Bellman equation. Theorem 3.1 is the main tool for proving next result that characterizes $\vartheta$ as a solution, in viscosity sense, of a HJB equation with oblique derivative boundary conditions. Set

$$
\bar{D}:=\left\{(x, y) \in \mathbb{R}^{d+1} \mid y \geq g(x)\right\}=\operatorname{Epigraph}(g),
$$

where $D$ is the interior of $\bar{D}$.

Theorem 3.2. Under assumptions (H1) and (H2), $\vartheta$ is a continuous viscosity solution of the following HJB equation

$$
\begin{array}{ll}
-\partial_{t} \vartheta+H\left(t, x, D_{x} \vartheta, D_{x}^{2} \vartheta\right)=0 & \text { in }[0, T) \times D \\
-\partial_{y} \vartheta=0 & \text { on }[0, T) \times \partial D \\
\vartheta(T, x, y)=\psi(x, y) & \text { in } \bar{D}
\end{array}
$$

with

$$
H(t, x, p, Q):=\sup _{u \in U}\left\{-b(t, x, u) p-\frac{1}{2} \operatorname{Tr}\left[\sigma \sigma^{T}\right](t, x, u) Q\right\} .
$$

(In all the paper $D_{x}, D_{x}^{2}$ denote respectively the first and second order derivatives with respect to $x$.)

Before starting the proof we recall the definition of viscosity solution for problem (3.5) (see [21] and the references therein for a complete discussion on weak boundary conditions).

Definition 3.3. A usc function $\bar{\vartheta}$ (resp. lsc function $\underline{\vartheta}$ ) on $[0, T] \times \bar{D}$ is a viscosity sub-solution (resp. super-solution) of (3.5), if for each function $\underline{\varphi} \in C^{1,2}([0, T] \times \bar{D})$, at each maximum (resp. minimum) point $(t, x, y)$ of $\bar{\vartheta}-\varphi($ resp. $\underline{\vartheta}-\varphi)$ the following inequalities hold

$$
\begin{cases}-\partial_{t} \varphi+H\left(t, x, D_{x} \varphi, D_{x}^{2} \varphi\right) \leq 0 & \text { in }[0, T) \times D \\ \min \left(-\partial_{t} \varphi+H\left(t, x, D_{x} \varphi, D_{x}^{2} \varphi\right),-\partial_{y} \varphi\right) \leq 0 & \text { on }[0, T) \times \partial D \\ \min \left(\bar{\vartheta}-\psi,-\partial_{t} \varphi+H\left(t, x, D_{x} \varphi, D_{x}^{2} \varphi\right),-\partial_{y} \varphi\right) \leq 0 & \text { on }\{T\} \times \partial D \\ \min \left(\bar{\vartheta}-\psi,-\partial_{t} \varphi+H\left(t, x, D_{x} \varphi, D_{x}^{2} \varphi\right)\right) \leq 0 & \text { on }\{T\} \times D\end{cases}
$$

(resp.

$$
\begin{cases}-\partial_{t} \varphi+H\left(t, x, D_{x} \varphi, D_{x}^{2} \varphi\right) \geq 0 & \text { in }[0, T) \times D \\ \max \left(-\partial_{t} \varphi+H\left(t, x, D_{x} \varphi, D_{x}^{2} \varphi\right),-\partial_{y} \varphi\right) \geq 0 & \text { on }[0, T) \times \partial D \\ \max \left(\underline{\vartheta}-\psi,-\partial_{t} \varphi+H\left(t, x, D_{x} \varphi, D_{x}^{2} \varphi\right),-\partial_{y} \varphi\right) \geq 0 & \text { on }\{T\} \times \partial D \\ \max \left(\underline{\vartheta}-\psi,-\partial_{t} \varphi+H\left(t, x, D_{x} \varphi, D_{x}^{2} \varphi\right)\right) \geq 0 & \text { on }\{T\} \times D .)\end{cases}
$$

Finally a continuous function $\vartheta$ is a viscosity solution of 3.5 if it is both a sub- and a super-solution.

Proof of Theorem 3.2. First, from the definition of $\vartheta$, we obtain easily that $\vartheta(T, x, y)=\psi(x, y)$.

Now, we check that $\vartheta$ is a viscosity sub-solution. Let $\varphi \in C^{1,2}([0, T] \times \bar{D})$ such that $\vartheta-\varphi$ attains a maximum at point $(\bar{t}, \bar{x}, \bar{y}) \in[0, T) \times \bar{D}$. Without 
loss of generality we can always assume that $(\bar{t}, \bar{x}, \bar{y})$ is a strict local maximum point (let us say in a ball of radius $r>0$ centered in $(\bar{t}, \bar{x}, \bar{y})$ ) and $\vartheta(\bar{t}, \bar{x}, \bar{y})=$ $\varphi(\bar{t}, \bar{x}, \bar{y})$. Thanks to Theorem 3.1, for any $u \in \mathcal{U}$ and for any sufficiently small stopping time $\theta=\theta^{u}$, we have:

$$
\begin{aligned}
\varphi(\bar{t}, \bar{x}, \bar{y}) & =\vartheta(\bar{t}, \bar{x}, \bar{y}) \leq \mathbb{E}\left[\vartheta\left(\theta, X_{\bar{t}, \bar{x}}^{u}(\theta), Y_{\bar{t}, \bar{x}, \bar{y}}^{u}(\theta)\right)\right] \\
& \leq \mathbb{E}\left[\varphi\left(\theta, X_{\bar{t}, \bar{x}}^{u}(\theta), Y_{\bar{t}, \bar{x}, \bar{y}}^{u}(\theta)\right)\right] .
\end{aligned}
$$

Two cases will be considered depending on if the point $(\bar{x}, \bar{y})$ belongs or not to the boundary of $D$.

- Case 1: $g(\bar{x})<\bar{y}$. Consider a constant control $u(s) \equiv u \in U$. From the continuity of $g$ and the a.s. continuity of the sample paths it follows that, for a.e. $\omega \in \Omega$, there exists $\bar{s}(\omega)>\bar{t}$ such that $g\left(X_{\bar{t}, \bar{x}}^{u}(s)\right)<\bar{y}$ if $s \in[\bar{t}, \bar{s}(\omega))$.

Let be $h>0$, and let $\bar{\theta}$ be a the following stopping time:

$\bar{\theta}:=\inf \left\{s>\bar{t} \mid\left(X_{\bar{t}, \bar{x}}^{u}(s), Y_{\bar{t}, \bar{x}, \bar{y}}^{u}(s)\right) \notin B_{r}(\bar{x}, \bar{y})\right\} \wedge(\bar{t}+h) \wedge \inf \left\{s>\bar{t} \mid g\left(X_{\bar{t}, \bar{x}}^{u}(s)\right) \geq \bar{y}\right\}$,

(here and through the paper $B_{r}\left(x_{0}\right)$ denotes the ball of radius $r>0$ centered at $\left.x_{0}\right)$. One can easily observe that a.s. $Y_{\bar{t}, \bar{x}, \bar{y}}^{u}(\bar{\theta})=\bar{y}$, then by 3.9

$$
\varphi(\bar{t}, \bar{x}, \bar{y}) \leq \mathbb{E}\left[\varphi\left(\bar{\theta}, X_{\bar{t}, \bar{x}}^{u}(\bar{\theta}), \bar{y}\right)\right] \quad \forall u \in U .
$$

By applying the Ito's formula, and thanks to the smoothness of $\varphi$, we get:

$$
\mathbb{E}\left[\int_{\bar{t}}^{\bar{\theta}}-\partial_{t} \varphi-b\left(s, X_{\bar{t}, \bar{x}}^{u}(s), u\right) D_{x} \varphi-\frac{1}{2} \operatorname{Tr}\left[\sigma \sigma^{T}\right]\left(s, X_{\bar{t}, \bar{x}}^{u}(s), u\right) D_{x}^{2} \varphi d s\right] \leq 0 .
$$

Since the stopping times

$\inf \left\{s>\bar{t} \mid\left(X_{\bar{t}, \bar{x}}^{u}(s), Y_{\bar{t}, \bar{x}, \bar{y}}^{u}(s)\right) \notin B_{r}(\bar{x}, \bar{y})\right\} \quad$ and $\quad \inf \left\{s>\bar{t} \mid g\left(X_{\bar{t}, \bar{x}}^{u}(s)\right) \geq \bar{y}\right\}$ are a.s. strictly greather than $\bar{t}$, it follows that a.s. $\bar{\theta}-\bar{t}>0$. Therefore,

$\mathbb{E}\left[\frac{1}{\bar{\theta}-\bar{t}} \int_{\bar{t}}^{\bar{\theta}}-\partial_{t} \varphi-b\left(s, X_{\bar{t}, \bar{x}}^{u}(s), u\right) D_{x} \varphi-\frac{1}{2} \operatorname{Tr}\left[\sigma \sigma^{T}\right]\left(s, X_{\bar{t}, \bar{x}}^{u}(s), u\right) D_{x}^{2} \varphi d s\right] \leq 0$.

By the dominate convergence theorem and the mean value theorem, letting $h$ going to 0 , it follows

$$
-\partial_{t} \varphi-b(\bar{t}, \bar{x}, u) D_{x} \varphi-\frac{1}{2} \operatorname{Tr}\left[\sigma \sigma^{T}\right](\bar{t}, \bar{x}, u) D_{x}^{2} \varphi \leq 0, \quad \forall u \in U,
$$

and finally:

$$
-\partial_{t} \varphi+\sup _{u \in U}\left\{-b(\bar{t}, \bar{x}, u) D_{x} \varphi-\frac{1}{2} \operatorname{Tr}\left[\sigma \sigma^{T}\right](\bar{t}, \bar{x}, u) D_{x}^{2} \varphi\right\} \leq 0 .
$$

- Case 2: $g(\bar{x})=\bar{y}$. Assume that $-\partial_{y} \varphi(\bar{t}, \bar{x}, \bar{y})>0$, otherwise the conclusion is straightforward. Consider a constant control $u(s) \equiv u \in U$. Thanks to the continuity of the sample paths and the smoothness of $\varphi$, for a.e. $\omega$ there is a time $\bar{s}(\omega)>\bar{t}$ and $\eta>0$ such that:

$$
\varphi\left(s, X_{\bar{t}, \bar{x}}^{u}(s), y\right) \leq \varphi\left(s, X_{\bar{t}, \bar{x}}^{u}(s), \bar{y}\right) \quad \forall s \in[\bar{t}, \bar{s}], y \in[\bar{y}, \bar{y}+\eta) .
$$


Let $\bar{\theta}$ be the stopping time given by:

$$
\begin{aligned}
\bar{\theta} & :=\inf \left\{s>\bar{t} \mid\left(X_{\bar{t}, \bar{x}}^{u}(s), Y_{\bar{t}, \bar{x}, \bar{y}}^{u}(s)\right) \notin B_{r}(\bar{x}, \bar{y})\right\} \wedge \inf \left\{s>\bar{t} \mid g\left(X_{\bar{t}, \bar{x}}^{u}(s)\right) \notin[\bar{y}, \bar{y}+\eta)\right\} \\
& \wedge \inf \left\{s>\bar{t} \mid \partial_{y} \varphi\left(s, X_{\bar{t}, \bar{x}}^{u}(s), \bar{y}\right) \geq 0\right\} \wedge(\bar{t}+h) .
\end{aligned}
$$

By (3.9) one has

$$
\varphi(\bar{t}, \bar{x}, \bar{y}) \leq \mathbb{E}\left[\varphi\left(\bar{\theta}, X_{\bar{t}, \bar{x}}^{u}(\bar{\theta}), \bar{y}\right)\right]
$$

which implies:

$$
-\partial_{t} \varphi+\sup _{u \in U}\left\{-b(\bar{t}, \bar{x}, u) D \varphi-\frac{1}{2} \operatorname{Tr}\left[\sigma \sigma^{T}\right](\bar{t}, \bar{x}, u) D^{2} \varphi\right\} \leq 0 .
$$

In conclusion,

$$
\min \left(-\partial_{t} \varphi+\sup _{u \in U}\left\{-b(\bar{t}, \bar{x}, u) D \varphi-\frac{1}{2} \operatorname{Tr}\left[\sigma \sigma^{T}\right](\bar{t}, \bar{x}, u) D^{2} \varphi\right\},-\partial_{y} \varphi\right) \leq 0,
$$

and $\vartheta$ is a viscosity sub-solution of equation 3.5.

It remains to prove that $\vartheta$ is a viscosity super-solution of $(3.5)$. Let $\varphi \in C^{1,2}([0, T] \times \bar{D})$ be such that $\vartheta-\varphi$ attains a minimum at point $(\bar{t}, \bar{x}, \bar{y}) \in$ $[0, T) \times \bar{D}$. Without loss of generality we can always assume that $(\bar{t}, \bar{x}, \bar{y})$ is a strict local minimum point and $\vartheta(\bar{t}, \bar{x}, \bar{y})=\varphi(\bar{t}, \bar{x}, \bar{y})$. Thanks to the DPP, for any sufficiently small stopping time $\theta=\theta^{u}$ one has

$$
\begin{aligned}
\varphi(\bar{t}, \bar{x}, \bar{y}) & =\vartheta(\bar{t}, \bar{x}, \bar{y})=\inf _{u \in \mathcal{U}} \mathbb{E}\left[\vartheta\left(\theta, X_{\bar{t}, \bar{x}}^{u}(\theta), Y_{\bar{t}, \bar{x}, \bar{y}}^{u}(\theta)\right)\right] \\
& \geq \inf _{u \in \mathcal{U}} \mathbb{E}\left[\varphi\left(\theta, X_{\bar{t}, \bar{x}}^{u}(\theta), Y_{\bar{t}, \bar{x}, \bar{y}}^{u}(\theta)\right)\right] .
\end{aligned}
$$

This can be treated by using again the same arguments as for the sub-solution proof.

3.3. Comparison principle. The section is concluded with a comparison principle for equation (3.5). There is a large literature dealing with Neumann-type or oblique derivative boundary conditions. We refer to [34, 9 ] for the first order case and to [29, 28, for second order equations. For dealing with this kind of problems some regularity assumption on the domain $D$ is often required. In our case the definition of $D$ is strictly connected with the choice of the function $g$ that is, without further hypothesis, only Lipschitz continuous. The result below has the advantage of taking into account also this non smooth case.

Theorem 3.4. Assume that (H1)-(H2) are satisfied. Let $\bar{\vartheta}$ (resp. 므) a usc (resp. lsc) viscosity sub-solution (resp. super-solution) of (3.5) satisfying the following growth condition (for $C>0$ ):

$$
\begin{aligned}
\bar{\vartheta}(t, x, y) & \leq C(1+|x|+|y|), \\
\text { (resp. } \underline{\vartheta}(t, x, y) & \geq-C(1+|x|+|y|) .
\end{aligned}
$$

Then $\bar{\vartheta} \leq \underline{\vartheta}$ on $[0, T] \times \bar{D}$. 
Such a comparison principle is proved in 25] for elliptic equations in bounded domains. The arguments extend the ones used in [29, 28] for the case when the domain $D$ has a smooth boundary. For convenience of the reader we give the main steps of the proof in Appendix A, and show how the arguments can be adapted for the parabolic case in unbounded domains. Assertions of Theorems 3.2 and 3.4 lead to the following result:

Corollary 3.5. Under assumptions (H1) and (H2), the value function $\vartheta$ is the unique continuous, Lipschitz in $(x, y)$, viscosity solution of equation (3.5) on $[0, T] \times \bar{D}$.

The uniqueness result is stated on $\bar{D}$, which means that the solution to the HJB equation coincides with the value function $\vartheta$ on $[0, T] \times \bar{D}$. Then $\vartheta$ is extended in a unique way on $[0, T] \times \mathbb{R}^{d} \times \mathbb{R}$ by: $\vartheta(t, x, y)=\vartheta(t, x, y \vee g(x))$.

\section{NUMERICAL APPROXIMATION}

Now, we focus on general approximation schemes for (3.5). Let $N \geq 1$ be an integer (number of time steps), and let

$$
h:=\frac{T}{N}, \quad \text { and } \quad t_{n}:=n h,
$$

for $n=0, \ldots, N$. Let $\Delta x=\left(\Delta x_{1}, \ldots, \Delta x_{d}\right) \in\left(\mathbb{R}_{+}^{*}\right)^{d}$ be a mesh step in $\mathbb{R}^{d}$, $\Delta y>0$ be a mesh step in $\mathbb{R}$, and $\rho:=(h, \Delta x, \Delta y)$ be a set of mesh steps (in time and space).

For a given $\rho$, consider the corresponding space grid

$$
\mathcal{G}_{\rho}:=\left\{\left(x_{i}, y_{j}\right)=(i \Delta x, j \Delta y), \text { for }(i, j) \in \mathbb{Z}^{d} \times \mathbb{Z}\right\} .
$$

where for $i \in \mathbb{Z}^{d}, i \Delta x:=\left(i_{1} \Delta x_{1}, \cdots, i_{N} \Delta x_{N}\right)$. For any $x \in \mathbb{R}^{d}$, let $j_{x} \in \mathbb{Z}$ be the upper integer part of $\frac{g(x)}{\Delta y}$, i.e,

$$
j_{x}:=\min \{j \in \mathbb{Z}, j \Delta y \geq g(x)\} .
$$

Consider a projection operator (along the direction $e_{y}$ ) $\Pi^{\mathcal{G}_{\rho}}$ defined as follows:

$$
\Pi^{\mathcal{G}_{\rho}}(\Phi)\left(t_{n}, x_{i}, y_{j}\right):= \begin{cases}\Phi\left(t_{n}, x_{i}, y_{j}\right), & \forall j \geq j_{x_{i}}, i \in \mathbb{Z}^{d} \\ \Phi\left(t_{n}, x_{i}, y_{j_{x_{i}}}\right), & \forall j<j_{x_{i}}, i \in \mathbb{Z}^{d}\end{cases}
$$

for any $\Phi \in C\left([0, T] \times \mathbb{R}^{d} \times \mathbb{R}, \mathbb{R}\right)$.

We aim at computing an approximation $W_{i, j}^{n}$ of the value function $\vartheta\left(t_{n}, x_{i}, y_{j}\right)$, on the grid $\mathcal{G}_{\rho}$. By $W^{\rho}$, we will denote the interpolation of the values $W_{i, j}^{n}$ at $\left(t_{n}, x_{i}, y_{j}\right)$. The values $W_{i, j}^{n}$ are defined recusively as follows:

\section{General scheme (GS)}

1)Define $W_{i, j}^{N}:=\psi\left(x_{i}, y_{j} \vee g\left(x_{i}\right)\right), \forall i, j$. 
2)For $n=N, \ldots, 1$, the value $W_{i, j}^{n-1}$ is obtained as solution of:

$$
\begin{aligned}
& S^{\rho}\left(t_{n-1}, x_{i}, y_{j}, W_{i, j}^{n-1}, \Pi^{\mathcal{G}_{\rho}}\left(W^{\rho}\right)\right)=0, \quad \forall i, j \text { with } g\left(x_{i}\right) \leq y_{j}, \\
& W_{i, j}^{n-1}:=W_{i, j_{i}}^{n-1}, \quad \forall i, j \text { with } g\left(x_{i}\right)>y_{j},
\end{aligned}
$$

where $S^{\rho}:[0, T] \times \bar{D} \times \mathbb{R} \times C\left([0, T] \times \mathbb{R}^{d} \times \mathbb{R}, \mathbb{R}\right) \rightarrow \mathbb{R}$ is a scheme operator.

Typical operator $S^{\rho}$ can be obtained by using an explicit or implicit finite difference method on (3.5a) (see [16, 15, 33]), or a semi-Lagrangian (SL) scheme ([35, 19, 24]). In (4.1), the value of $W_{i, j}^{n-1}$ may depend on the whole function $W^{\rho}$ (i.e., all the values $W_{i, j}^{k}$ for $\left.k=0, \cdots, N\right)$. Of course, in case of an explicit time discretization, the step (4.1) could be re-written as:

$$
S^{\rho}\left(t_{n-1}, x_{i}, y_{j}, W_{i, j}^{n-1}, \Pi^{\mathcal{G}_{\rho}}\left(W^{\rho}\left(t_{n}, \cdot, \cdot\right)\right)\right)=0, \quad \forall i, j \text { with } g\left(x_{i}\right) \leq y_{j} .
$$

However, the formulation (4.1) is more general and includes different kind of time-discretization like Euler implicit scheme or $\theta$-methods.

The main idea of the numerical method described here above is to mix the use of a standard scheme for (3.5a), together with a "projection step" on $\partial D$ in order to approximate the homogeneous oblique derivative boundary condition (3.5b). Let us point out that a similar method was introduced in [5] for the case $g(x) \equiv|x|$. However, the general case with possibly nonlinear function $g$ requires some further attention on the boundary.

4.1. A general convergence result. In this part we closely follow the arguments of Barles and Souganidis [10], using consistency, stability and monotonicity arguments. For this, we assume that the following hypotheses are considered:

(H3) (stability) For any $\rho$, there exists a solution $W^{\rho}$ of the scheme which has linear growth (uniformly in $\rho$ ), that is, there exists a constant $C>0$, independent of $\rho$, such that

$$
\left|W^{\rho}(t, x, y)\right| \leq C(1+|x|+|y|), \quad \text { on } \quad[0, T] \times \bar{D}
$$

(H4) (consistency) The scheme $S^{\rho}$ is consistent with respect to equation (3.5a) in $[0, T] \times \bar{D}$, that is, for all $(t, x, y) \in[0, T] \times \bar{D}$ and for every $\Phi \in C^{1,2}([0, T] \times \bar{D})$,

$$
\lim _{\substack{\rho \rightarrow 0 \\[0, T] \times \bar{D} \ni(s, \xi, \gamma) \rightarrow(t, x, y) \\ \zeta \rightarrow 0}} S^{\rho}(s, \xi, \gamma, \Phi(s, \xi, \gamma)+\zeta, \Phi+\zeta)=-\partial_{t} \Phi+H\left(t, x, D_{x} \Phi, D_{x}^{2} \Phi\right) .
$$

(H5) (monotonicity) For any $\rho$, for $r \in \mathbb{R},(t, x, y) \in[0, T] \times \bar{D}, S^{\rho}(t, x, y, r, \Phi)$ depends only on the values of $\Phi$ in a neighborhood $B_{\eta(\rho)}(t, x, y)$ of $(t, x, y)$, with $\eta(\rho) \geq 0$ such that $\eta(\rho) \stackrel{\rho \rightarrow 0}{\longrightarrow} 0$. Moreover, for any $\Phi_{1}, \Phi_{2}$ functions on $[0, T] \times \mathbb{R}^{d} \times \mathbb{R} \rightarrow \mathbb{R}$, such that $\Phi_{1} \geq \Phi_{2}$ on $B_{\eta(\rho)}((t, x, y))$, it holds:

$$
S^{\rho}\left(t, x, y, r, \Phi_{1}\right) \leq S^{\rho}\left(t, x, y, r, \Phi_{2}\right) .
$$


Let us point out that the monotonicity and the consistency are required here only for the operator scheme $S^{\rho}$ that corresponds to the discretization of the equation $-\partial_{t} \vartheta+H\left(t, x, D \vartheta, D^{2} \vartheta\right)=0$.

Notice also that the monotonicity assumption (H5) is slightly different from the classical one usualy required for general HJB equations. The reason for this comes from the fact that the operator scheme $S^{\rho}$ is defined on $\bar{D}$ and may use some values of the function $W^{\rho}$ outside the domain $D$ that are obtained by oblique projection through the projection step. Our monotonicity assumption requires local dependency on the values of $\Phi$ in a neighborhood of the point under consideration, then a comparison of the scheme values is requested for any two functions $\Phi_{1}$ and $\Phi_{2}$ such that $\Phi_{1} \geq \Phi_{2}$ only on this neighborhood. The proof of Theorem 4.1 highlights what this requirement is needed for.

In the next section, we will check that assumptions (H3)-(H5) are well satisfied by the semi-Lagrangian scheme. Now, we state the convergence result for any general scheme satisfying (H3)-(H5).

Theorem 4.1. Assume (H1)-(H2). Let $S^{\rho}$ be a scheme satisfying (H3)(H5). Then, when $\rho$ tends to $0, W^{\rho}$ converges to the unique viscosity solution of (3.5) uniformly on each compact subset of $[0, T] \times \bar{D}$.

Proof. Let us define

$$
\begin{aligned}
\bar{W}(t, x, y) & :=\limsup _{[0, T] \times \bar{D} \ni(s, \xi, \gamma) \rightarrow(t, x, y)} W^{\rho}(s, \xi, \gamma) \\
\text { and } \quad \underline{W}(t, x, y) & :=\liminf _{\substack{[0, T] \times \bar{D} \ni(s, \xi, \gamma) \rightarrow(t, x, y) \\
\rho \rightarrow 0}} W^{\rho}(s, \xi, \gamma) .
\end{aligned}
$$

We start by proving that $\bar{W}$ is a viscosity sub-solution of equation (3.5) (the proof that $\underline{W}$ is a viscosity super-solution is analogous).

Let $\varphi$ be in $C^{1,2}([0, T] \times \bar{D})$ and let $(\bar{t}, \bar{x}, \bar{y})$ be a local maximum point for $\bar{W}-\varphi$ on $[0, T] \times \bar{D}$. Without loss of generality we can assume that $(\bar{t}, \bar{x}, \bar{y})$ is a strict local maximum in the restricted ball $B_{r}(\bar{t}, \bar{x}, \bar{y}) \cap([0, T] \times \bar{D})$ for a certain $r>0$,

$$
(\bar{W}-\varphi)(\bar{t}, \bar{x}, \bar{y})=\max _{B_{r} \cap([0, T] \times \bar{D})}(\bar{W}-\varphi)=0,
$$

and $\varphi \geq 2 \sup _{\rho}\left\|W^{\rho}\right\|_{\infty}$ outside the ball $B_{r}(\bar{t}, \bar{x}, \bar{y})$.

We first assume that $\bar{t} \in(0, T)$. Then we claim that:

$$
\begin{aligned}
& -\partial_{t} \varphi+H\left(\bar{t}, \bar{x}, D_{x} \varphi, D_{x}^{2} \varphi\right) \leq 0 \quad \text { if }(\bar{x}, \bar{y}) \in D,(4.4 \mathrm{a}) \\
& \text { or } \min \left(-\partial_{t} \varphi+H\left(\bar{t}, \bar{x}, D_{x} \varphi, D_{x}^{2} \varphi\right),-\partial_{y} \varphi\right) \leq 0 \text { if }(\bar{x}, \bar{y}) \in \partial D \text {. (4.4b) }
\end{aligned}
$$

Following [21, there exists a sequence of mesh steps $\rho_{k}$ and a sequence $\left(t_{k-1}, x_{k}, y_{k}\right)$ in $(0, T) \times \bar{D}$ such that: $\rho_{k} \rightarrow 0$ and $\left(t_{k-1}, x_{k}, y_{k}\right) \rightarrow(\bar{t}, \bar{x}, \bar{y})$ as 
$k \rightarrow+\infty$, and $\left(t_{k-1}, x_{k}, y_{k}\right)$ is a global maximum point of $W^{\rho_{k}}-\varphi$, with

$$
\left(W^{\rho_{k}}-\varphi\right)\left(t_{k-1}, x_{k}, y_{k}\right)=\max _{[0, T] \times \bar{D}}\left(W^{\rho_{k}}-\varphi\right)=\delta_{k} \stackrel{k \rightarrow \infty}{\longrightarrow} 0
$$

and

$$
W^{\rho_{k}}\left(t_{k-1}, x_{k}, y_{k}\right) \stackrel{k \rightarrow \infty}{\longrightarrow} \bar{W}(\bar{t}, \bar{x}, \bar{y}) .
$$

- Case 1: assume that $\bar{y}>g(\bar{x})$. Thus, $(\bar{x}, \bar{y})$ is in the open set $D$ and for $k$ large enough, $\left(x_{k}, y_{k}\right) \in D$. By continuity of $g, y>g(x)$ in $B_{\eta\left(\rho_{k}\right)}\left(t_{k-1}, x_{k}, y_{k}\right)$ for $\rho_{k}$ small enough. Therefore $\Pi^{\mathcal{G}_{\rho}}\left(W^{\rho_{k}}\right)=W^{\rho_{k}} \leq$ $\varphi+\delta_{k}$ on $B_{\eta\left(\rho_{k}\right)}\left(t_{k-1}, x_{k}, y_{k}\right)$. On the other hand, $W^{\rho_{k}}\left(t_{k-1}, x_{k}, y_{k}\right)=$ $\varphi\left(t_{k-1}, x_{k}, y_{k}\right)+\delta_{k}$. Hence, thanks to the monotonicity of the scheme, it follows

$$
\begin{aligned}
0 & =S^{\rho_{k}}\left(t_{k-1}, x_{k}, y_{k}, W^{\rho_{k}}\left(t_{k-1}, x_{k}, y_{k}\right), \Pi^{\mathcal{G}_{\rho}}\left(W^{\rho_{k}}\right)\right) \\
& \geq S^{\rho_{k}}\left(t_{k-1}, x_{k}, y_{k}, \varphi\left(t_{k-1}, x_{k}, y_{k}\right)+\delta_{k}, \varphi+\delta_{k}\right) .
\end{aligned}
$$

Using the consistency of the scheme, we obtain in the limit when $\rho_{k} \rightarrow 0$,

$$
-\partial_{t} \varphi+H\left(\bar{t}, \bar{x}, D_{x} \varphi, D_{x}^{2} \varphi\right) \leq 0 .
$$

We conclude that $4.4 \mathrm{a}$ is satisfied when $(\bar{x}, \bar{y}) \in D$.

- Case 2: when $\bar{y}=g(\bar{x}),\left(x_{k}, y_{k}\right)$ can be also on $\partial D$ and the scheme may involve values $W_{k \ell}^{n}$ on some points $\left(x_{k}, y_{\ell}\right)$ that are not in $\bar{D}$. Here, we need to consider two sub-cases.

- Sub-Case 2.1: if $-\partial_{y} \varphi(\bar{t}, \bar{x}, \bar{y}) \leq 0$, then 4.4b holds.

- Sub-Case 2.2: if $-\partial_{y} \varphi(\bar{t}, \bar{x}, \bar{y})>0$, then there exists a neighborhood $\mathcal{V}$ of $(\bar{t}, \bar{x}, \bar{y})$ where $\partial_{y} \varphi(t, x, y)$ is well defined, and $-\partial_{y} \varphi(t, x, y)>0$ for every $(t, x, y) \in \mathcal{V}$. Therefore,

$$
y \leq y^{\prime} \Longrightarrow \varphi(t, x, y) \geq \varphi\left(t, x, y^{\prime}\right) \quad \forall(t, x, y),\left(t, x, y^{\prime}\right) \in \mathcal{V} .
$$

For $k$ large enough, $B_{\eta\left(\rho_{k}\right)}\left(t_{k-1}, x_{k}, y_{k}\right) \subset \mathcal{V}$. Let $(t, x, y) \in B_{\eta\left(\rho_{k}\right)}\left(t_{k-1}, x_{k}, y_{k}\right)$. If $y \geq g(x)$, then

$$
\Pi^{\mathcal{G}_{\rho}}\left(W^{\rho_{k}}(t, x, y)\right)=W^{\rho_{k}}(t, x, y) \leq \varphi(t, x, y)+\delta_{k} .
$$

Otherwise if $y<g(x), \Pi^{\mathcal{G}_{\rho}}\left(W^{\rho_{k}}(t, x, y)\right)=W^{\rho_{k}}\left(t, x, y_{j_{x}}\right)$, and we have

$$
\begin{aligned}
\Pi^{\mathcal{G}_{\rho}}\left(W^{\rho_{k}}(t, x, y)\right) & =W^{\rho_{k}}\left(t, x, y_{j_{x}}\right) \\
& \leq \varphi\left(t, x, y_{j_{x}}\right)+\delta_{k} \quad \text { (by using 4.5) } \\
& \leq \varphi(t, x, y)+\delta_{k} \quad \text { (by using 4.7) }
\end{aligned}
$$

(for 4.10) we also used the continuity of $g$ that ensures that for $k$ big enough $\left(t, x, y_{j_{x}}\right)$ still lays in $\left.\mathcal{V}\right)$. We conclude $\Pi^{\mathcal{G}_{\rho}}\left(W^{\rho_{k}}\right) \leq \varphi+\delta_{k}$ on $B_{\eta\left(\rho_{k}\right)}\left(t_{k-1}, x_{k}, y_{k}\right)$. Thus by monotonicity (H5), we get

$$
\begin{aligned}
0 & =S^{\rho_{k}}\left(t_{k-1}, x_{k}, y_{k}, W^{\rho_{k}}\left(t_{k-1}, x_{k}, y_{k}\right), \Pi^{\mathcal{G}_{\rho}}\left(W^{\rho_{k}}\right)\right) \\
& \geq S^{\rho_{k}}\left(t_{k-1}, x_{k}, y_{k}, \varphi\left(t_{k-1}, x_{k}, y_{k}\right)+\delta_{k}, \varphi+\delta_{k}\right) .
\end{aligned}
$$

Using the consistency of the scheme, when $\rho_{k} \rightarrow 0$, we get

$$
-\partial_{t} \varphi+H\left(\bar{t}, \bar{x}, D_{x} \varphi, D_{x}^{2} \varphi\right) \leq 0 .
$$


As conclusion for case $2,4.4 \mathrm{~b}$ is satisfied when $(\bar{x}, \bar{y}) \in \partial D$.

Similar arguments can be used in order to treat the case $\bar{t}=T$ (classical arguments enable to treat also the case of the boundary $\bar{t}=0$ ). We conclude that $\bar{W}$ is a viscosity sub-solution of the equation (3.5).

As already mentioned, we can also show that $\underline{W}$ is a super-solution. Then by the comparison principle on $[0, T] \times \bar{D}$ (Theorem 3.4 and using the growth condition (4.3)), the inequality $\underline{W} \geq \bar{W}$ holds on $[0, T] \times \bar{D}$. Furthermore since the reverse inequality $\underline{W} \leq \bar{W}$ is always true, we deduce that $\bar{W}=$ $\underline{W}=\vartheta$ on $[0, T] \times \bar{D}$. Hence the convergence result is proved.

4.2. A semi-Lagrangian scheme. We first introduce some new notations: for any $u \in U$, the $\operatorname{drift} b(\cdot, \cdot, u)$ (resp. volatility $\sigma(\cdot, \cdot, u)$ ) will be simply denoted by $b^{u}$ (resp. $\left.\sigma^{u}\right)$.

Consider the operator $\mathcal{T}: C\left(\mathbb{R}^{d+1}\right) \rightarrow C\left([0, T] \times \mathbb{R}^{d+1}\right)$ defined by:

$$
\mathcal{T}(\phi)(t, x, y)=\min _{u \in U} \frac{1}{2 p} \sum_{k=1}^{2 p}[\phi]_{x}\left(x+h b^{u}(t, x)+\sqrt{h p}(-1)^{k} \sigma_{\left\lfloor\frac{k+1}{2}\right\rfloor}^{u}(t, x), y\right)
$$

where $\left(\sigma_{k}^{u}\right)_{k=1, \ldots, p}$ are the column vectors of $\sigma^{u}$, and $\lfloor q\rfloor$ denotes the integer part of $q$. The notation $[\cdot] \equiv[\cdot]_{x}$ stands for a monotone, $P_{1}$ interpolation operator on the $x$-grid $\left(x_{i}\right)$, satisfying for every Lipschitz continuous function $\phi$ (with Lipschitz constant $L_{\phi}$ ):

$$
\left\{\begin{array}{l}
(i)[\phi]_{x}\left(x_{i}\right)=\phi\left(x_{i}\right), \forall i \\
(i i)\left|[\phi]_{x}(x)-\phi(x)\right| \leq L_{\phi}|\Delta x|, \\
(\text { iii })\left|[\phi]_{x}(x)-\phi(x)\right| \leq C|\Delta x|^{2}\left\|D_{x}^{2} \phi\right\|_{\infty} \text { if } \phi \in C^{2}\left(\mathbb{R}^{d}\right), \\
\text { (iv) for any functions } \phi_{1}, \phi_{2}: \mathbb{R}^{d} \rightarrow \mathbb{R}, \phi_{1} \leq \phi_{2} \Rightarrow\left[\phi_{1}\right]_{x} \leq\left[\phi_{2}\right]_{x} .
\end{array}\right.
$$

The operator $\mathcal{T}$ corresponds to a discretisation of the equation $3.5 \mathrm{a}$ by a semi-Lagrangian scheme (see [35, 19, 24]). Now, define an approximation method for the system (3.5) as follows:

Fully discrete scheme (FDS)

1)Initialisation step: for all $i, j$, set $W_{i, j}^{N}=\psi\left(x_{i}, y_{j} \vee g\left(x_{i}\right)\right)$.

2)For $n=N, \ldots, 1$ :

- Step 1 Compute $W_{i, j}^{n-\frac{1}{2}}=\mathcal{T}\left(W_{., .}^{n}\right)\left(t_{n}, x_{i}, y_{j}\right)$, for all $i, j$ (here $W_{\cdot, \text {, }}^{n}$ denotes the values $\left.\left\{W_{i, j}^{n} \mid i, j \in \mathbb{Z}^{d} \times \mathbb{Z}\right\}\right)$;

- Step 2 Compute $W_{i, j}^{n-1}=\Pi^{\mathcal{G}^{\rho}}\left(W^{n-\frac{1}{2}}\right)$, for all $i, j$.

The FDS scheme is a particular GS method, with the following formula for the operator $S^{\rho}$ :

$$
S^{\rho}(t-h, x, y, r, \Phi):=\frac{1}{h}\left\{r-\Pi^{\mathcal{G}^{\rho}}(\mathcal{T}(\Phi)(t, x, y))\right\} .
$$

Remark 4.2. We point out that in the FDS, for $j$ such that $y_{j}<g\left(x_{i}\right)$, the projection step fixes the value of $W_{i, j}^{n-1}$ as $W_{i, j_{x_{i}}}^{n-1}$. Of course, other alternatives could be considered, for instance, one could consider the scheme: 
1) Initialization step: for $n=N$, for all $i, j$, set $\widetilde{W}_{i, j}^{N}=\psi\left(x_{i}, y_{j} \vee g\left(x_{i}\right)\right)$.

2) For $n=N, \ldots, 1, \widetilde{W}_{i, j}^{n-1}=\widetilde{\mathcal{T}}\left(\widetilde{W}_{.,}^{n}\right)\left(t_{n}, x_{i}, y_{j}\right)$, for all $i, j$, where

$$
\widetilde{\mathcal{T}}(\phi)(t, x, y)=\min _{u \in U} \frac{1}{2 p} \sum_{k=1}^{2 p}[\phi]_{x, y}\left(x+h b^{u}(t, x)+\sqrt{h p}(-1)^{k} \sigma_{\left\lfloor\frac{k+1}{2}\right\rfloor}^{u}(t, x), y \vee g(x)\right),
$$

with $[\cdot]_{x, y}$ standing for an interpolation in both variables $x$ and $y$.

All the convergence results stated in the paper also hold for this new scheme.

In the next subsection, we will study some properties of the approximated solution $W^{\rho}$. Before this, we define also the following semi-discrete scheme where we consider only a time-discretization:

\section{Semi-discrete scheme (SDS)}

1)For $n=N$, for every $(x, y) \in \mathbb{R}^{d+1}$, set $V^{N}(x, y)=\psi(x, y \vee g(x))$.

2)For $n=N, \ldots, 1$, define $V^{n-1}$ as the function defined by:

$$
V^{n-1}(x, y)=\mathcal{T}_{0}\left(V^{n}\right)\left(t_{n}, x, y \vee g(x)\right),
$$

where, $\mathcal{T}_{0}$ is defined from $C\left(\mathbb{R}^{d+1}\right)$ into $C\left([0, T] \times \mathbb{R}^{d+1}\right)$ by:

$$
\mathcal{T}_{0}(\phi)(t, x, y):=\min _{u \in U} \frac{1}{2 p} \sum_{k=1}^{2 p} \phi\left(x+h b^{u}(t, x)+(-1)^{k} \sqrt{h p} \sigma_{\left\lfloor\frac{k+1}{2}\right\rfloor}^{u}(t, x), y\right) .
$$

Unlike the fully discrete scheme, no interpolation step is required in the SDS. Straightforward calculations lead to the following consistency estimate, for any $\phi \in C^{2,4}\left((0, T) \times \mathbb{R}^{d} \times \mathbb{R}\right)$ :

$$
\begin{aligned}
& \left|\frac{1}{h}\left(\phi(t-h, x, y)-\mathcal{T}_{0}(\phi)(t, x, y)\right)-\left(-\partial_{t} \phi+H\left(t, x, D_{x} \phi, D_{x}^{2} \phi\right)\right)\right| \\
& \leq K_{1} \max _{u \in U}\left(\left\|\partial_{t t}^{2} \phi\right\|_{\infty}+\left|b^{u}(t, x)\right|^{2}\left\|D_{x}^{2} \phi\right\|_{\infty}+\left|b^{u}(t, x)\left\|\left.\sigma^{u}(t, x)\right|^{2}\right\| D_{x}^{3} \phi \|_{\infty}\right.\right. \\
& \left.\quad+\left|\sigma^{u}(t, x)\right|^{4}\left\|D_{x}^{4} \phi\right\|_{\infty}\right) h,
\end{aligned}
$$

for any $(x, y) \in \mathbb{R}^{d}$, with $K_{1}$ a positive constant independent of $\phi$. Moreover, $\mathcal{T}$ and $\mathcal{T}_{0}$ satisfy the following properties :

Lemma 4.3. For every $\phi_{1}, \phi_{2} \in C\left([0, T] \times \mathbb{R}^{d} \times \mathbb{R}\right)$, we have:

(i) $\left(\mathcal{T}_{0}\left(\phi_{1}\right)-\mathcal{T}_{0}\left(\phi_{2}\right)\right)_{+} \leq\left\|\left(\phi_{1}-\phi_{2}\right)_{+}\right\|$;

(ii) $\left\|\mathcal{T} \phi_{1}-\mathcal{T} \phi_{2}\right\|_{\infty} \leq\left\|\phi_{1}-\phi_{2}\right\|_{\infty}$;

(iii) $\left\|\mathcal{T}_{0} \phi_{1}-\mathcal{T}_{0} \phi_{2}\right\|_{\infty} \leq\left\|\phi_{1}-\phi_{2}\right\|_{\infty}$;

(iv) for any Lipschitz continuous function $\phi$ on $\mathbb{R}^{d} \times \mathbb{R},\left\|\mathcal{T} \phi-\mathcal{T}_{0} \phi\right\|_{\infty} \leq$ $L_{\phi} \Delta x$, where $L_{\phi}$ is a Lipschitz constant of $\phi$.

Notice that assertion $(i)$ corresponds to a discrete comparison principle. The proof of the lemma is straightforward and will be omitted. 
4.3. Convergence of the SL scheme. The aim of this subsection is to prove a convergence result for the scheme FDS.

Theorem 4.4. Let assumptions (H1)-(H2) be satisfied. Let $W^{\rho}$ be defined by the fully discrete scheme. Assume also that

$$
\frac{|(\Delta x, \Delta y)|}{h} \leq C \text { (where } C \text { is a positive constant) }
$$

Then the FDS is convergent, i.e. $W^{\rho}$ converges towards $\vartheta$, as $\rho$ tends to 0 , uniformly on each compact set of $[0, T] \times \bar{D}$.

The claim of this theorem would be a consequence of the general result of theorem 4.1, provided the monotonicity, consistency and stability properties of the FDS are established. The monotonicity and the consistency are easy to check. If $\psi$ is a bounded function, the stability of the scheme would be immediate because in this case $\left\|W^{n}\right\|_{\infty} \leq\|\psi\|_{\infty}$. However, in the general case the function $\psi$ is not necessarily bounded. In order to derive the stability property, we first prove some results on the semi-discrete scheme that we state in the following next two propositions.

Proposition 4.5. If assumptions (H1) and (H2) are satisfied, the solution $V$ of the semi-discrete scheme is Lipschitz continuous in $(x, y)$ and $\frac{1}{2}$-Hölder continuous in $t$ : there exists a constant $L_{V} \geq 0$, for every $0 \leq n, m \leq N$, and every $\left(x_{1}, y_{1}\right),\left(x_{2}, y_{2}\right) \in \mathbb{R}^{d+1}$ such that

$\left|V^{n}\left(x_{2}, y_{2}\right)-V^{m}\left(x_{1}, y_{1}\right)\right| \leq L_{V}\left(\left|x_{2}-x_{1}\right|+\left|y_{2}-y_{1}\right|+\left(1+\left|x_{1}\right|\right)\left|t_{n}-t_{m}\right|^{1 / 2}\right)$.

Proof. Let $\left(\xi^{n}, \xi^{n+1}, \ldots, \xi^{N-1}\right)$, be independent random variables, with values in $\{0, \ldots, 2 p\}$, and such that $\mathbb{P}\left(\xi^{n}=k\right)=\frac{1}{2 p}, \quad \forall k=1, \ldots, 2 p$.

Let $u=\left(u_{n}, u_{n+1}, \ldots, u_{N-1}\right)$ denotes a sequence of controls, with $u_{i} \in U$, and let $Z_{k}^{n}=Z_{k}^{n, x, u}, n \leq k \leq N$, be the random walk such that:

$$
\left\{\begin{array}{l}
Z_{n}^{n}=x, \\
Z_{k+1}^{n}=Z_{k}^{n}+h b^{u_{k}}\left(t_{k+1}, Z_{k}^{n}\right)+\sqrt{h} \bar{\sigma}_{\xi^{k}}^{u_{k}}\left(t_{k+1}, Z_{k}^{n}\right), k \geq n
\end{array}\right.
$$

where the notation $\bar{\sigma}_{k}^{u}(t, x):=(-1)^{k} \sqrt{p} \sigma_{\left\lfloor\frac{k+1}{2}\right\rfloor}^{u}(t, x)$ is used. Notice that $Z_{k}^{n, x, u}$ depends only on the controls $\left(u_{n}, \ldots, u_{k-1}\right)$. Direct calculations lead to the following expressions:

$$
\begin{aligned}
V^{n}(x, y) & =\min _{u_{n} \in U} \frac{1}{2 p} \sum_{k=1}^{2 p} V^{n+1}\left(x+h b^{u_{n}}\left(t_{n+1}, x\right)+\sqrt{h} \bar{\sigma}_{k}^{u_{n}}\left(t_{n}, x\right), y \vee g(x)\right) \\
& =\min _{u_{n} \in U} \mathbb{E}\left[V^{n+1}\left(Z_{n+1}^{n, x, u}, y \vee g(x)\right)\right],
\end{aligned}
$$


and, in the same way,

$$
\begin{aligned}
V^{n}(x, y) & =\min _{u_{n} \in U} \mathbb{E}\left[\min _{u_{n+1} \in U} \mathbb{E}\left[V^{n+2}\left(Z_{n+2}^{n+1, Z_{n+1}^{n, x, u}, u}, y \vee g(x) \vee g\left(Z_{n+1}^{n, x, u}\right)\right)\right]\right] \\
& =\min _{u_{n} \in U} \mathbb{E}\left[\min _{u_{n+1} \in U} \mathbb{E}\left[V^{n+2}\left(Z_{n+2}^{n, x, u}, y \vee g(x) \vee g\left(Z_{n+1}^{n, x, u}\right)\right)\right]\right],
\end{aligned}
$$

and so on, and finally (also using $\left.V^{N}(x, y) \equiv \psi(x, y \vee g(x))\right)$ :

$$
\begin{aligned}
V^{n}(x, y) & =\min _{u_{n}} \mathbb{E}\left[\min _{u_{n+1}} \mathbb{E}\left[\cdots \min _{u_{N-1}} \mathbb{E}\left[V^{N}\left(Z_{N}^{n, x, u}, y \vee \max _{i=n, \ldots, N-1} g\left(Z_{i}^{n, x, u}\right)\right)\right]\right] \cdots\right] \\
& =\min _{u_{n}} \mathbb{E}\left[\min _{u_{n+1}} \mathbb{E}\left[\cdots \min _{u_{N-1}} \mathbb{E}\left[\psi\left(Z_{N}^{n, x, u}, y \vee \max _{i=n, \ldots, N} g\left(Z_{i}^{n, x, u}\right)\right)\right]\right] \cdots\right] .
\end{aligned}
$$

Then, we have the equivalent representation formula:

$$
V^{n}(x, y)=\min _{u=\left(u_{n}, \ldots, u_{N-1}\right)} \mathbb{E}\left[\psi\left(Z_{N}^{n, x, u}, y \vee \max _{i=n, \ldots, N} g\left(Z_{i}^{n, x, u}\right)\right)\right] .
$$

By using $\left|\left(x_{2} \vee y_{2}\right)-\left(x_{1} \vee y_{1}\right)\right| \leq\left|x_{1}-x_{2}\right| \vee\left|y_{1}-y_{2}\right| \leq\left|x_{1}-x_{2}\right|+\left|y_{1}-y_{2}\right|$, we obtain:

$$
\begin{aligned}
& \left|V^{n}\left(x_{2}, y_{2}\right)-V^{n}\left(x_{1}, y_{1}\right)\right| \leq \\
& \quad \leq L_{\psi} \max _{u} \mathbb{E}\left[\left|Z_{N}^{n, x_{2}, u}-Z_{N}^{n, x_{1}, u}\right|+L_{g} \max _{n \leq i \leq N}\left|Z_{i}^{n, x_{2}, u}-Z_{i}^{n, x_{1}, u}\right|+L_{g}\left|y_{2}-y_{1}\right|\right] \\
& \quad \leq L_{\psi}\left(L_{g}+1\right)\left(\max _{u} \mathbb{E}\left[\max _{n \leq i \leq N}\left|Z_{i}^{n, x_{2}, u}-Z_{i}^{n, x_{1}, u}\right|\right]+\left|y_{2}-y_{1}\right|\right)
\end{aligned}
$$

where max denotes the maximum over $\left(u_{n}, u_{n+1}, \ldots, u_{N-1}\right) \in U^{N-n}$. By (H1), there exists a constant $C_{1} \geq 0$, that depends only on $\left(T, L_{\sigma}, L_{b}\right)$, such that for every $0 \leq m, n \leq N$ and every $x, x_{1}, x_{2} \in \mathbb{R}^{d}$, the following classical estimates hold:

$$
\begin{aligned}
& \mathbb{E}\left[\max _{n \leq i \leq N}\left|Z_{i}^{n, x_{2}, u}-Z_{i}^{n, x_{1}, u}\right|\right] \leq C_{1}\left|x_{2}-x_{1}\right| \\
& \mathbb{E}\left[\max _{0 \leq i \leq p}\left|Z_{m+i}^{n, x, u}-Z_{m}^{n, x, u}\right|\right] \leq C_{1}\left|t_{m+p}-t_{m}\right|^{1 / 2}(1+|x|)
\end{aligned}
$$

Combining (4.20) and 4.21a), for $n=m$, the Lipschitz property follows:

$$
\left|V^{n}\left(x_{1}, y_{1}\right)-V^{n}\left(x_{2}, y_{2}\right)\right| \leq L_{\psi}\left(L_{g}+1\right)\left(C_{1}\left|x_{2}-x_{1}\right|+\left|y_{2}-y_{1}\right|\right) .
$$

On the other hand, for $0 \leq n \leq m \leq N$ by using again (4.19) we have:

$$
\left|V^{m}(x, y)-V^{n}(x, y)\right| \leq C \max _{u} \mathbb{E}\left[\left|\max _{n \leq i \leq N} Z_{i}^{n, x, u}-\max _{m \leq i \leq N} Z_{i}^{m, x, u}\right|\right]
$$


for some constant $C$. Therefore, since $Z_{m}^{m, x, u}=x$ :

$$
\begin{aligned}
&\left|V^{m}(x, y)-V^{n}(x, y)\right| \leq C \max _{u} \mathbb{E}\left[\left|\max _{n \leq i \leq m} Z_{i}^{n, x, u}-x\right|\right] \\
&+C \max _{u} \mathbb{E}\left[\left|\max _{m+1 \leq i \leq N} Z_{i}^{n, x, u}-\max _{m+1 \leq i \leq N} Z_{i}^{m, x, u}\right|\right] .
\end{aligned}
$$

The right term of $(4.23)$ is bounded by $C(1+|x|)\left|t_{m}-t_{n}\right|^{1 / 2}$. In order to bound (4.24), by using that $Z_{i}^{n, x, u}=Z_{i}^{m, Z_{m}^{n, x, u}, u}(\forall i \geq m)$ and 4.21a) the following estimate is obtained:

$$
\begin{aligned}
\mathbb{E}\left[\left|\max _{m+1 \leq i \leq N} Z_{i}^{m, Z_{m}^{n, x, u}, u}-\max _{m+1 \leq i \leq N} Z_{i}^{m, x, u}\right|\right] & \leq C_{1} \mathbb{E}\left[Z_{m}^{n, x, u}-x\right] \\
& \leq C_{1}^{2}(1+|x|)\left|t_{m}-t_{n}\right|^{1 / 2} .
\end{aligned}
$$

Hence it holds, for some constant $C$,

$$
\left|V^{n}(x, y)-V^{m}(x, y)\right| \leq C(1+|x|)\left|t_{m}-t_{n}\right|^{1 / 2} .
$$

Together with the Lipschitz property the desired result follows.

Remark 4.6. It is not clear whether the solution $W^{\rho}$ obtained by the fully discrete scheme satisfies a Lipschitz continuity property or not. The main difficulty is that a representation formula as 4.19 is not guaranteed for $W^{\rho}$.

Proposition 4.7. If assumptions (H1) and (H2) are satisfied, there exists a constant $C>0$ independent of $\rho$ such that, $\forall n=0, \ldots, N$ :

$$
\left\|W^{n}-V^{n}\right\|_{\infty} \leq C \frac{|(\Delta x, \Delta y)|}{h} .
$$

Proof. Consider the operator $\Pi: C\left(\mathbb{R}^{d} \times \mathbb{R}\right) \rightarrow C\left(\mathbb{R}^{d} \times \mathbb{R}\right)$ defined by $\Pi \phi(x, y)=\phi(x, y \vee g(x))$. With this notation, we have $V^{n-1}=\Pi\left(\mathcal{T}_{0}\left(V^{n}\right)\right)$.

On the other hand, $W_{i j}^{n-1}=\Pi^{\mathcal{G}^{\rho}}\left(\mathcal{T}\left(W^{n}\right)\left(t_{n}, x_{i}, y_{j}\right)\right)$. Therefore, by using Lemma 4.3 , we get:

$$
\begin{aligned}
\left\|W^{n-1}-V^{n-1}\right\|_{\infty} & \leq\left\|\Pi^{\mathcal{G}^{\rho}}\left(\mathcal{T}\left(W^{n}\right)\right)-\Pi\left(\mathcal{T}_{0}\left(V^{n}\right)\right)\right\|_{\infty} \\
& \leq\left\|\Pi^{\mathcal{G}^{\rho}}\left(\mathcal{T}\left(W^{n}\right)\right)-\Pi^{\mathcal{G}^{\rho}}\left(\mathcal{T}_{0}\left(V^{n}\right)\right)\right\|_{\infty}+\left\|\Pi^{\mathcal{G}^{\rho}}\left(\mathcal{T}_{0}\left(V^{n}\right)\right)-\Pi\left(\mathcal{T}_{0}\left(V^{n}\right)\right)\right\|_{\infty} \\
& \leq\left\|\mathcal{T}\left(W^{n}\right)-\mathcal{T}_{0}\left(V^{n}\right)\right\|_{\infty}+\left\|\Pi^{\mathcal{G}^{\rho}}\left(\mathcal{T}_{0}\left(V^{n}\right)\right)-\Pi\left(\mathcal{T}_{0}\left(V^{n}\right)\right)\right\|_{\infty} \\
& \leq\left\|\mathcal{T}\left(W^{n}\right)-\mathcal{T}\left(V^{n}\right)\right\|_{\infty}+\left\|\mathcal{T}\left(V^{n}\right)-\mathcal{T}_{0}\left(V^{n}\right)\right\|_{\infty}+L_{V} \Delta y \\
& \leq\left\|W^{n}-V^{n}\right\|_{\infty}+L_{V}|\Delta x|+L_{V} \Delta y .
\end{aligned}
$$

By recursion, it follows:

$$
\left\|W^{n}-V^{n}\right\|_{\infty} \leq\left\|W^{N}-V^{N}\right\|_{\infty}+n L_{V}(|\Delta x|+\Delta y) .
$$

Since $V^{N}(x, y)=\psi(x, y \vee g(x))$ on $\mathbb{R}^{d+1}, W^{N}$ is an interpolation of $V^{N}$ on the grid $\mathcal{G}^{\rho}$ and $n \leq \frac{T}{h}$, we obtain the desired result.

Now we turn to the proof of Theorem 4.4 
Proof of Theorem 4.4. Since $V^{n}$ is a Lipschitz function in the $(x, y)$ variables, uniformly in $n$, then $V^{n}$ has a linear growth with constants independent of $n$. By Lemma 4.7, and by using assumption (4.17),

$$
\left\|W^{n}-V^{n}\right\|_{\infty} \leq C \frac{|(\Delta x, \Delta y)|}{h} \leq K .
$$

Hence $W^{n}$ has also a linear growth, with constants independent of $n$.

Moreover, the consistency of the scheme comes from the estimate 4.16) and using that $\frac{|\Delta x|^{2}}{h} \rightarrow 0$ by assumption 4.17). Also, the monotonicity property (as defined in (H5)) is deduced from the monotonicity of the interpolations and the monotonicity of the operator $\mathcal{T}_{0}$.

In consequence, the convergence result follows from Theorem 4.1 .

Remark 4.8. The convergence result of Theorem 4.4 is still valid in the case where $\psi$ is a bounded Lipschitz function and $\frac{|\Delta x|^{2}}{h} \rightarrow 0$.

\section{ERROR BOUNDS FOR THE SEMI-LAGRANGIAN SCHEME}

In the sequel, for any function $f$ defined from some set $Q \subset(0, \infty) \times \mathbb{R}^{d+1}$ into either $\mathbb{R}, \mathbb{R}^{d}$ or some space of matrices, we set

$$
[f]_{1}:=\sup _{(t, x) \neq(s, y)} \frac{|f(t, x)-f(s, y)|}{\left(|x-y|+|t-s|^{1 / 2}\right)}
$$

and

$$
|f|_{1}:=\|f\|_{\infty}+[f]_{1} .
$$

In this section we aim to give an error bound for the fully discrete scheme FDS, as well as the semi-discrete SL scheme (4.15). For this aim a supplementary hypothesis will be required (together with $(\mathrm{H} 1)$ and $(\mathrm{H} 2)$ ):

(H1') There is a constant $M_{1}$ independent of $u \in \mathcal{U}$ such that:

$$
\left|\sigma^{u}\right|_{1}+\left|b^{u}\right|_{1} \leq M_{1} .
$$

The error estimates theory is based on some technique of "shaking coefficients" and regularization introduced by Krylov in [30, 31] and studied later by many authors [6, 7, 8, 13, 14, 24]. The main idea consists of regularizing the exact solution $\vartheta$ in order to obtain a smooth sub-solution $\vartheta_{\varepsilon}$ (for an approximation parameter $\varepsilon>0$ ) to the equation (3.5), then the upper-bound error estimate can be obtained by using the consistency estimate (4.16). The regularisation procedure takes advantage from the fact that the exact solution $\vartheta$ is Hölder continuous, which enables to obtain an estimate of $\left|\vartheta-\vartheta_{\varepsilon}\right|_{\infty}$ of order $O(\varepsilon)$. The lower bound is obtained by similar arguments, but in this case we need to build a smooth sub-solution to the discrete equation. For this, a regularization of the numerical solution will be considered. However, as noticed earlier (remark 4.6), it is not clear if the solution $W^{\rho}$ of the fully discrete scheme is Hölder continuous or not, and then it is not clear how to build directly a regularization $W_{\varepsilon}^{\rho}$ (with regularisation parameter $\varepsilon$ ) with an error $\left|W^{\rho}-W_{\varepsilon}^{\rho}\right|$ of order $O(\varepsilon)$. For this reason, we will start by deriving an 
error estimate between the solution $\vartheta$ and the solution $V$ of the semi-discrete scheme SDS, using the fact that $V$ has Hölder continuity properties. Then the estimates for the FDS are derived as a consequence of Lemma 4.7.

For the regularization procedure, consider a smooth function $\mu: \mathbb{R}^{d+2} \rightarrow$ $\mathbb{R}$, with $\mu \geq 0$, supported in $(0,1) \times B_{1}(0)$, with $\int_{\mathbb{R}} \int_{\mathbb{R}^{d}} \mu(s, x) d x d s=1$, and define $\mu_{\varepsilon}$ as the following sequence of mollifiers:

$$
\mu_{\varepsilon}(t, x, y):=\frac{1}{\varepsilon^{d+3}} \mu\left(\frac{t}{\varepsilon^{2}}, \frac{x}{\varepsilon}, \frac{y}{\varepsilon}\right) \quad \text { in } \mathbb{R} \times \mathbb{R}^{d+1} .
$$

5.1. The rate of convergence for the semi-discrete scheme. For any $\varepsilon>0$, let $\mathcal{E}$ be the set of progressively measurable processes $(\alpha, \chi)$ valued in $\left[-\varepsilon^{2}, 0\right] \times B(0, \varepsilon) \subset \mathbb{R} \times \mathbb{R}^{d}$ that is,

$$
\mathcal{E}:=\{\text { prog. meas. process }(\alpha, \chi) \text { valued in } E\},
$$

where $E:=\left\{(a, e) \in \mathbb{R} \times \mathbb{R}^{d},-\varepsilon^{2} \leq a \leq 0,|e| \leq \varepsilon\right\}$. by

On other hand, let $\bar{M}:=2 \sqrt{1+L_{g}^{2}}$ and introduce $g_{\varepsilon}: \mathbb{R}^{d} \rightarrow \mathbb{R}$ defined

$$
g_{\varepsilon}(x):=g(x)-\bar{M} \varepsilon .
$$

Finally, let us denote by $D^{\varepsilon}$ the set defined as follows:

$$
D^{\varepsilon}:=\left\{(x, y) \in \mathbb{R}^{d+1}, y>g_{\varepsilon}(x)\right\} .
$$

Remark 5.1. The choice of $g_{\varepsilon}$ is such that the following property holds:

$$
(x, y) \in \bar{D} \Longrightarrow\left(x-e_{1}, y-e_{2}\right) \in D^{\varepsilon} \quad \forall\left(e_{1}, e_{2}\right) \in \mathbb{R}^{d+1},\left|\left(e_{1}, e_{2}\right)\right| \leq \varepsilon .
$$

5.1.1. Upper bound. Now, we start by introducing a perturbed control problem (with "shaking coefficients"). For any $\varepsilon>0$, consider the following value function

$$
\vartheta^{\varepsilon}(t, x, y):=\inf _{\substack{u \in \mathcal{U},(\alpha, \chi) \in \mathcal{E}}} \mathbb{E}\left[\psi\left(X_{t, x}^{u,(\alpha, \chi)}(T), \max _{s \in[t, T]} g_{\varepsilon}\left(X_{t, x}^{u,(\alpha, \chi)}(s)\right) \vee y\right)\right],
$$

where $X_{t, x}^{u,(\alpha, \chi)}(\cdot)$ is the solution of the perturbed system of SDEs

$$
\left\{\begin{array}{l}
d X(s)=b(s+\alpha(s), X(s)+\chi(s), u(s)) d s+\sigma(s+\alpha(s), X(s)+\chi(s), u(s)) d \mathcal{B}(s) \\
X(t)=x .
\end{array}\right.
$$

Remark 5.2. The functions $\sigma$ and $b$ are only defined for times $t \in[0, T]$, but they can be extended to times $\left[-2 \varepsilon^{2}, T+2 \varepsilon^{2}\right]$ in such a way that assumption (H1') still holds.

Proposition 5.3. Under assumptions (H1)-(H2) and (H1') (extending eventually $b$ and $\sigma$ as prescribed in Remark 5.2), the following holds: 
(i) $\vartheta^{\varepsilon}$ is a Lipschitz continuous function in $x$ and $y$ and $a \frac{1}{2}$-Hölder continuous function in $t$. More precisely, for $t, \leq t^{\prime} \in[0, T], x, x^{\prime} \in$ $\mathbb{R}^{d}$ and $y, y^{\prime} \in \mathbb{R}$, we have:

$\left|\vartheta^{\varepsilon}(t, x, y)-\vartheta^{\varepsilon}\left(t^{\prime}, x^{\prime}, y^{\prime}\right)\right| \leq L_{\vartheta}\left(\left|x-x^{\prime}\right|+\left|y-y^{\prime}\right|+(1+|x|)\left|t-t^{\prime}\right|^{\frac{1}{2}}\right)$.

(ii) $\left|\vartheta(t, x, y)-\vartheta^{\varepsilon}(t, x, y)\right| \leq C \varepsilon$ on $[0, T] \times \bar{D}$, where the constant $C$ only depends on $T$ and the Lipschitz constants of $b, \sigma, g$ and $\psi$.

Proof. The Lipschitz and Hölder continuity follows by the same arguments as in Proposition 2.2. Thanks to the Lipschitz continuity of $\psi$ and $g$ and the choice of $g_{\varepsilon}$ one has

$$
\left|\vartheta(t, x, y)-\vartheta^{\varepsilon}(t, x, y)\right| \leq K\left(\sup _{\substack{u \in \mathcal{U},(\alpha, \chi) \in \mathcal{E}}} \mathbb{E}\left[\sup _{s \in[t, T]}\left|X_{t, x}^{u}(s)-X_{t, x}^{u,(\alpha, \chi)}(s)\right|\right]+\bar{M} \varepsilon\right)
$$

and the result is obtained by classical estimates thanks to the hypothesis on $b$ and $\sigma$.

Theorem 5.4. Let assumptions (H1)-(H2) and (H1') be satisfied. There exists a constant $C \geq 0$ such that

$$
\vartheta\left(t_{n}, x, y\right)-V^{n}(x, y) \leq C h^{\frac{1}{4}}
$$

for all $n \geq 0$ (with $n h \leq T)$ and $(x, y) \in \bar{D}$.

Proof. The proof is splitted into three steps:

- Step 1. Let $\left(\tilde{a},\left(\tilde{e}_{1}, \tilde{e}_{2}\right)\right) \in \mathbb{R} \times \mathbb{R}^{d+1}$ be such that $-\varepsilon^{2} \leq \tilde{a} \leq 0$ and $\left|\left(\tilde{e}_{1}, \tilde{e}_{2}\right)\right| \leq \varepsilon$. Let $(\bar{t}, \bar{x}, \bar{y}) \in(0, T) \times D$, and define for $\nu>0$ :

$$
I_{\nu}(\bar{t}, \bar{x}, \bar{y}):=\left\{(t, x, y) \mid t \in\left[\bar{t}-\nu^{2}, \bar{t}\right],(x, y) \in B_{\nu}(\bar{x}, \bar{y})\right\} .
$$

We claim that $\vartheta^{\varepsilon}\left(\cdot-\tilde{a}, \cdot-\tilde{e}_{1}, \cdot-\tilde{e}_{2}\right)$ is a viscosity sub-solution of

$$
-\partial_{t} \vartheta+H\left(t, x, D_{x} \vartheta, D_{x}^{2} \vartheta\right) \leq 0 \quad \text { on } I_{\varepsilon}(\bar{t}, \bar{x}, \bar{y}) .
$$

To prove this claim, we notice first that $I_{\varepsilon}(\bar{t}, \bar{x}, \bar{y}) \subset D^{\varepsilon}$. Moreover, from Theorem 3.2 for any $\varepsilon>0, \vartheta^{\varepsilon}$ is a viscosity solution of the following equation

$$
\begin{cases}-\partial_{t} \vartheta^{\varepsilon}+\sup _{(a, e) \in E} H\left(t+a, x+e, D_{x} \vartheta^{\varepsilon}, D_{x}^{2} \vartheta^{\varepsilon}\right)=0 & \left(-2 \varepsilon^{2}, T\right) \times D^{\varepsilon}, \\ -\partial_{y} \vartheta^{\varepsilon}=0 & \left(-2 \varepsilon^{2}, T\right) \times \partial D^{\varepsilon}, \\ \vartheta^{\varepsilon}(T, x, y)=\psi(x,(g(x)-\bar{M} \varepsilon) \vee y) . & \end{cases}
$$

Let $\varphi \in C^{2,4}\left(\left[-2 \varepsilon^{2}, T\right] \times \overline{D^{\varepsilon}}\right)$ be such that $\vartheta^{\varepsilon}\left(\cdot-\tilde{a}, \cdot-\tilde{e}_{1}, \cdot-\tilde{e}_{2}\right)-\varphi$ achieves a local maximum at $(\tilde{t}, \tilde{x}, \tilde{y})$ on $I_{\varepsilon}(\bar{t}, \bar{x}, \bar{y})$. Clearly $\left(\tilde{t}-\tilde{a}, \tilde{x}-\tilde{e}_{1}, \tilde{y}-\tilde{e}_{2}\right)$ is also a local maximum of $\vartheta^{\varepsilon}-\varphi\left(\cdot+\tilde{a}, \cdot+\tilde{e}_{1}, \cdot+\tilde{e}_{2}\right)$ on $I_{2 \varepsilon}\left(\right.$ and $\left.I_{2 \varepsilon} \subset D^{\varepsilon}\right)$. Since $\vartheta^{\varepsilon}$ is a viscosity solution of equation (5.5), we obtain:

$$
-\partial_{t} \varphi(\tilde{t}, \tilde{x}, \tilde{y})+\sup _{\left(a, e_{1}\right) \in E} H\left(\tilde{t}-\tilde{a}+a, \tilde{x}-\tilde{e}_{1}+e_{1}, D_{x} \varphi, D_{x}^{2} \varphi\right) \leq 0 .
$$

Taking $\left(a, e_{1}\right)=\left(\tilde{a}, \tilde{e}_{1}\right)$, we get the result. 
- Step 2. Define following mollification $\vartheta_{\varepsilon}$ :

$\vartheta_{\varepsilon}(t, x, y):=\left(\vartheta^{\varepsilon} * \mu_{\varepsilon}\right)(t, x, y)=\int_{\substack{\left|\left(e_{1}, e_{2}\right)\right| \leq \varepsilon \\-\varepsilon^{2} \leq a \leq 0}} \vartheta^{\varepsilon}\left(t-a, x-e_{1}, y-e_{2}\right) \mu\left(a, e_{1}, e_{2}\right) d a d e$.

We recall the following properties of the mollifiers:

$$
\left|\vartheta^{\varepsilon}(t, x, y)-\vartheta_{\varepsilon}(t, x, y)\right| \leq\left[\vartheta^{\varepsilon}\right]_{1} \varepsilon
$$

with $\left[w^{\epsilon}\right]_{1} \leq C L_{\vartheta}, C \geq 0$. Moreover, for any $i \geq 1$ or $j \geq 1$,

$$
\left\|D_{x}^{i} \vartheta_{\varepsilon}\right\|_{\infty} \leq C L_{\vartheta} \varepsilon^{1-i}, \quad\left\|D_{t}^{j} \vartheta_{\varepsilon}\right\|_{\infty} \leq C L_{\vartheta} \varepsilon^{1-2 j}
$$

(where $D_{x}^{i}$ denotes the $i$-th derivative with respect to $x$, and $D_{t}^{j}$ the $j$-th derivative with respect to $t$ ). Since $\vartheta_{\varepsilon}$ is a limit of convex combinations of $\vartheta^{\varepsilon}\left(\cdot-\tilde{a}, \cdot-\tilde{e}_{1}, \cdot-\tilde{e}_{2}\right)$, then $\vartheta_{\varepsilon}$ satisfies in the viscosity sense

$$
-\partial_{t} \vartheta_{\varepsilon}+H\left(t, x, D_{x} \vartheta_{\varepsilon}, D_{x}^{2} \vartheta_{\varepsilon}\right) \leq 0 \quad \text { in }(0, T) \times D .
$$

Taking into account that $\vartheta_{\varepsilon}$ is in $C^{\infty}\left([0, T] \times \mathbb{R}^{d+1}\right)$, we conclude that (5.8) holds in classical sense on $[0, T] \times \bar{D}$. From the consistency estimate 4.16 along with (5.8) and by assumption (H1'), we get:

$$
\vartheta_{\varepsilon}\left(t_{n-1}, x, y\right)-\mathcal{T}_{0}\left(\vartheta_{\varepsilon}\right)\left(t_{n}, x, y\right) \leq C \frac{h^{2}}{\varepsilon^{3}} .
$$

Combining these bounds with Lemma 4.3, we get

$$
\begin{aligned}
\vartheta_{\varepsilon}\left(t_{n-1}, x, y\right)-V^{n-1}(x, y) & \leq \mathcal{T}_{0}\left(\vartheta_{\varepsilon}\right)\left(t_{n}, x, y\right)-\mathcal{T}_{0}\left(V^{n}\right)(x, y)+C \frac{h^{2}}{\varepsilon^{3}} \\
& \leq\left\|\left(\vartheta_{\varepsilon}\left(t_{n}, \cdot\right)-V^{n}\right)_{+}\right\|_{\infty}+C \frac{h^{2}}{\varepsilon^{3}} .
\end{aligned}
$$

Therefore, by recursion, it comes

$$
\left.\left\|\left(\vartheta_{\varepsilon}\left(t_{n}, \cdot\right)-V^{n}\right)_{+}\right\|_{\infty} \leq \|\left(\vartheta_{\varepsilon}(0, \cdot)-V^{N}\right)\right)_{+} \|_{\infty}+C T \frac{h}{\varepsilon^{3}} .
$$

- Step 3. By Proposition 5.3 together with the inequalities (5.6) and (5.9), we obtain for $n \geq 0$,

$$
\left\|\left(\vartheta\left(t_{n}, \cdot\right)-V^{n}\right)_{+}\right\|_{\infty} \leq C \varepsilon+C T \frac{h}{\varepsilon^{3}}, \quad n \geq 0 .
$$

The choice $\varepsilon^{4}=h$ leads to (for $n \geq 0$ ):

$$
\left\|\left(\vartheta\left(t_{n}, \cdot\right)-V^{n}\right)_{+}\right\|_{\infty} \leq C h^{\frac{1}{4}},
$$

which concludes the proof. 
5.1.2. Lower bound. For obtaining the lower bound we will apply exactly the same techniques as used for the upper bound, reversing the role of the equation and the scheme. The key point is that the solution $V$ of the semidiscrete scheme SDS is Lipschitz continuous. Then it is possible to use the techniques of "shaking coefficients" and regularisation to build a smooth sub-solution $V_{\varepsilon}$ satisying $\left\|V_{\varepsilon}^{n}-V^{n}\right\|_{\infty} \leq C \varepsilon$ and

$$
V_{\varepsilon}^{n-1}(x, y)-\mathcal{T}_{0}\left(V_{\varepsilon}^{n}\right)\left(t_{n}, x, y \vee g(x)\right) \leq 0 \quad \text { in }[0, T] \times \mathbb{R}^{d} \times \mathbb{R} .
$$

Then by consistency estimate and comparison principle, we conclude the following result:

Theorem 5.5. Let assumptions (H1)-(H2) and (H1') be satisfied. There exists a constant $C>0$ such that

$$
\vartheta\left(t_{n}, x, y\right)-V^{n}(x, y) \geq-C h^{\frac{1}{4}}
$$

for all $n \geq 0$ (with $N h \leq T)$ and $(x, y) \in \bar{D}$.

5.2. The fully discrete scheme. The section is concluded with the following theorem that provides error estimates for the fully discrete semiLagrangian scheme. The result is a simple consequence of Theorems 5.4, 5.5 and Lemma 4.7 .

Theorem 5.6. Let assumptions (H1)-(H2) and (H1') be satisfied. If $W$ is the solution of the fully-discrete scheme (4.14), there exists a constant $C>0$ independent from $n=0, \ldots, N$ such that

$$
\left\|W^{n}-\vartheta\left(t_{n}, \cdot\right)\right\|_{\infty} \leq C\left(h^{\frac{1}{4}}+\frac{|(\Delta x, \Delta y)|}{h}\right) .
$$

Proof. The result follows by using

$$
\left\|W^{n}-\vartheta\left(t_{n}, \cdot\right)\right\|_{\infty} \leq\left\|W^{n}-V^{n}\right\|_{\infty}+\left\|V^{n}-\vartheta\left(t_{n}, \cdot\right)\right\|_{\infty} .
$$

and thanks to Theorems 5.4, 5.5 and to Lemma 4.7 .

\section{Numerical tests}

In this section we present some numerical results for reachability problems in presence of state constraints (as in Section 3). The dynamics is given by the following controlled SDE in $\mathbb{R}^{2}$ :

$$
\left\{\begin{array}{l}
d X(s)=u(s)\left(\begin{array}{l}
1 \\
0
\end{array}\right) d s+u(s) \sigma(X(s)) d \mathcal{B}_{s}, s \geq t \\
X(t)=x
\end{array}\right.
$$

where $\mathcal{B}$ is a one-dimensional Brownian motion $(p=1), U=[0,1] \subset \mathbb{R}$ and the function $\sigma(x) \in \mathbb{R}^{2}$ will vary depending on the example. The target set is

$$
\mathcal{C}=\left\{x \equiv\left(x_{1}, x_{2}\right) \in \mathbb{R}^{2}, 0 \leq x_{1} \leq 0.4,\left|x_{2}\right| \leq 0.5\right\}
$$

and the set of state-constraints is

$$
\mathcal{K}=\mathbb{R}^{2} \backslash\left\{x \equiv\left(x_{1}, x_{2}\right) \in \mathbb{R}^{2},-0.4<x_{1}<-0.2,\left|x_{2}\right|<0.1\right\} .
$$


Given a final time $T$, the aim is to approximate the set $\mathcal{R}_{t}^{\mathcal{C}, \mathcal{K}}$ defined by (2.7), for $t=0$. We recall that the following auxiliary value is introduced:

$$
\vartheta(t, x, y)=\inf _{u \in \mathcal{U}} \mathbb{E}\left[d_{\mathcal{C}}\left(X_{t, x}^{u}(T)\right) \vee \max _{s \in[t, T]} d_{\mathcal{K}}\left(X_{t, x}^{u}(s)\right) \vee y\right]
$$

and that the characterization of the backward reachable set is given by

$$
x \in \mathcal{R}_{t}^{\mathcal{C}, \mathcal{K}} \Longleftrightarrow \vartheta(t, x, 0)=0 .
$$

In all the following tests, $t=0, T=0.5$ are fixed and the computational domain for $\left(x_{1}, x_{2}, y\right)$ is

$$
\left(x_{1}, x_{2}, y\right) \in \Omega=[-1,1] \times[-1,1] \times[0,1] .
$$

The numerical scheme implemented is the semi-Lagrangian scheme (FDS). We denote by $N_{t}$ the number of time-steps, $N_{x_{1}}=N_{x_{2}}$ and $N_{y}$ are the number of mesh steps for the space variables $\left(x_{1}, x_{2}, y\right)$, and

$$
h:=\frac{T}{N_{t}}, \quad \Delta x_{i}=\frac{2}{N_{x_{i}}}, \quad \Delta y=\frac{1}{N_{y}} .
$$

Different simulations show that the results are not very sensitive to the step discretization of the variable $y$. Unless otherwise precised, we set $N_{y}=$ 10 . For all tests, since $u \in[0,1]$ and because the dynamics depends linearly on the control $u$ it is sufficient to take only two controls $\left(N_{u}=2\right)$, e.g., $u \in\{0,1\}$ for the discretization of the control variable.

The different figures (see e.g. Figure 1) represent points in the $\left(x_{1}, x_{2}\right)$ plane. The obstacle is represented in black, and the target in dark grey. Then, an arbitrary non negative threshold $\epsilon:=10^{-5}$ is choosen, and our approximation of the reachable set is given by

$$
\widetilde{\mathcal{R}}_{t}^{\mathcal{C}, \mathcal{K}}:=\left\{\left(x_{1}, x_{2}\right) \in \mathbb{R}^{2}, \vartheta\left(0, x_{1}, x_{2}, \epsilon\right) \leq \epsilon\right\}
$$

(plotted in green).

Remark 6.1. This procedure is not very sensitive to the choice of $\epsilon$ in the range $10^{-2}-10^{-6}$.

Remark 6.2. We have also tested the scheme of Remark 4.2 and obtain similar results.

Test 1. In this test, there is no diffusion, that is:

$$
\sigma(x):=\left(\begin{array}{l}
0 \\
0
\end{array}\right) .
$$

Figure 1(a) shows the approximation obtained using $\left(N_{x_{1}}, N_{x_{2}}, N_{t}\right)=(800,800,200)$ (and the represented level set is $10^{-5}$ ).

The boundary of $\mathcal{R}_{t}^{\mathcal{C}, \mathcal{K}}$ (resp. $\widetilde{\mathcal{R}}_{t}^{\mathcal{C}, \mathcal{K}}$ ) is also represented by a black doted line (resp. red line). The result perfectly matches the expected solution. 


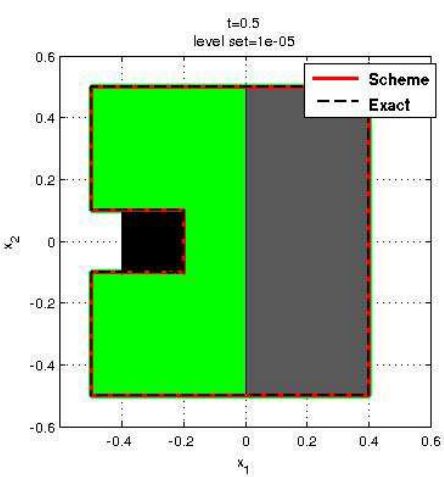

(a)

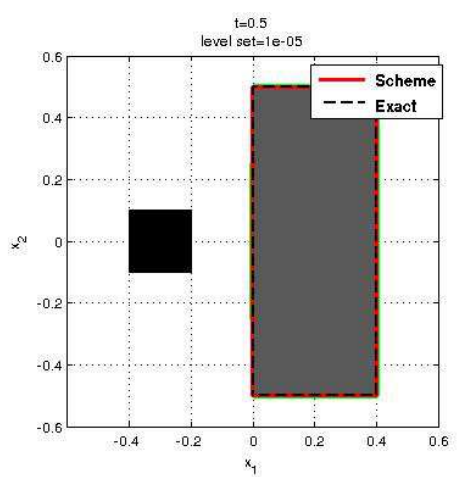

(b)

Figure 1. (a): Test 1, no diffusion, and (b): Test 2, with diffusion.

Test $\mathbf{2}$. In this test a non-zero volatility is considered:

$$
\sigma(x):=\left(\begin{array}{l}
0 \\
5
\end{array}\right)
$$

In that case the backward reachable set reduces to the target set, see Figure 1(b). In fact for any point outside the target, as soon as $u \neq 0$, even if the drift steers the system in the direction of the target, there is always a non-zero probability to go to far way in the $x_{2}$ orthogonal direction and therefore to not reach it.

Test 3. In this test the volatility is now given by

$$
\sigma(x)=5 d_{\Theta}(x)\left(\begin{array}{l}
0 \\
1
\end{array}\right)
$$

where $d_{\Theta}$ denotes the distance function to the set

$$
\Theta:=\left\{\left(x_{1}, x_{2}\right),\left|x_{2}\right| \geq 0.3\right\} .
$$

Hence for any point $\left(x_{1}, x_{2}\right)$, if $\left|x_{2}\right| \geq 0.3$ the volatility vanishes. According to the drift term, the target is finally reached, see Figure 2. This Figure shows the approximation of $\mathcal{R}_{t}^{\mathcal{C}, \mathcal{K}}$ for three different meshes. It also shows how the scheme converges. Notice that the points which are immediately above or below the obstacle are not in the reachable set since in presence of diffusion the state-constraint will be violated with a non-zero probability.

Also, in Table 1, various error norms are given to study the convergence of the scheme. For a given $n \geq 1$ we have chosen

$$
N_{x_{1}}=N_{x_{2}}=n, \quad N_{t}=n / 4 \quad \text { and } \quad N_{y}=n / 4 .
$$

Errors have been computed by taking $n=160$ for the reference value, and a convergence of order greater than one is observed in this simple example (better than the expected order of convergence). 

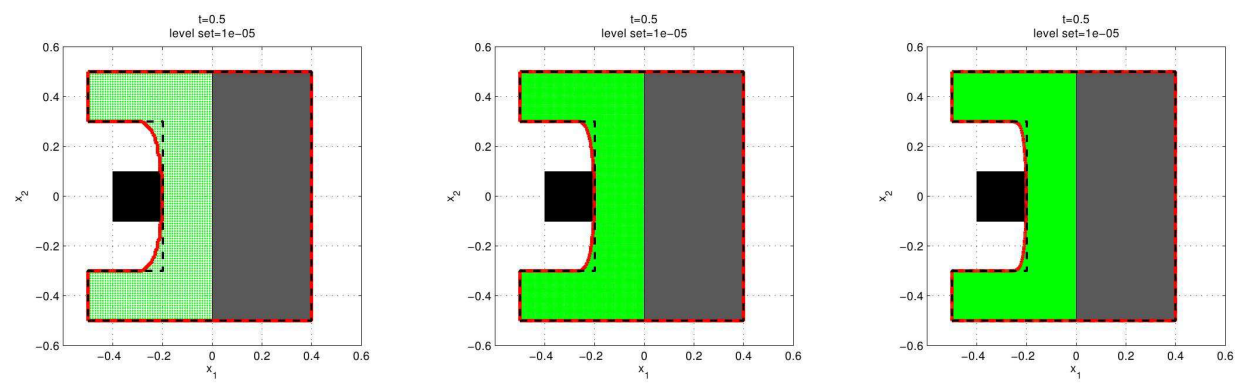

Figure 2. (Test 3) Vertical diffusion, using $\left(N_{x_{1}}, N_{x_{2}}, N_{t}\right)=(n, n, n / 4)$, for $n \in\{200,400,800\}$.

TABLE 1. Test 3, convergence table.

\begin{tabular}{|c|cc|cc|cc|}
\hline$n$ & $L^{\infty}$-error & order & $L^{1}$-error & order & $L^{2}$-error & order \\
\hline \hline 10 & 0.46582 & - & 0.02526 & - & 0.07115 & - \\
20 & 0.16633 & 1.48 & 0.00345 & 2.87 & 0.01979 & 1.84 \\
40 & 0.06746 & 1.30 & 0.00111 & 1.63 & 0.00668 & 1.56 \\
80 & 0.02500 & 1.43 & 0.00024 & 2.20 & 0.00194 & 1.78 \\
\hline
\end{tabular}

Test 4 (oblique diffusion) In this test the coefficient $\sigma$ is now given by

$$
\sigma(x)=5 d_{\Theta}(x)\left(\begin{array}{l}
1 \\
1
\end{array}\right)
$$

In Figure 3 we have plotted the results obtained with three different meshes, using $\left(N_{x_{1}}, N_{x_{2}}, N_{t}\right)=(n, n, n / 4)$ for $n \in\{100,200,400\}$. Altough the first computation plotted in Figure 3 (left, with $n=100$ ) is not very accurate, the other computations (with $n=200$ and $n=400$ ) clearly show the good convergence of the scheme.
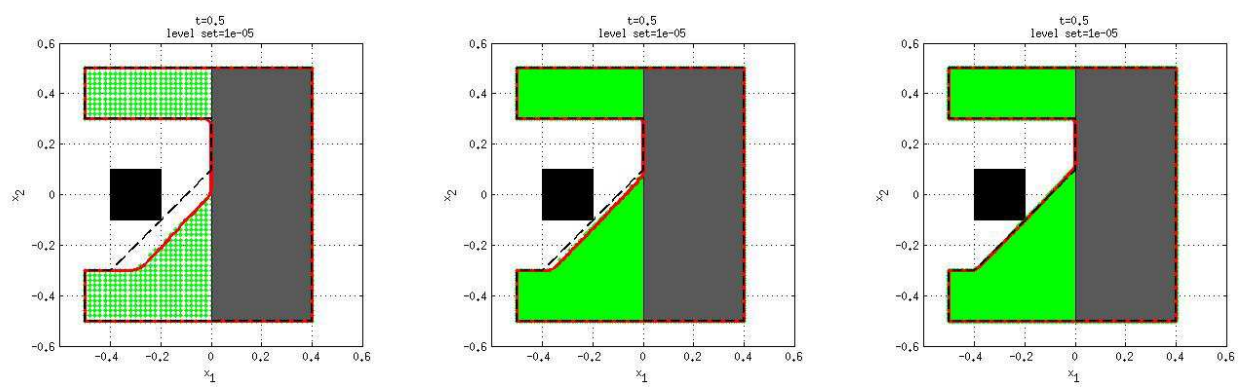

Figure 3. (Test 4) Oblique diffusion. using $\left(N_{x_{1}}, N_{x_{2}}, N_{t}\right)=(n, n, n / 4)$ for $n \in\{100,200,400\}$. 


\section{Appendix A. Proof of the Comparison principle}

In what follows we consider a general HJB equation with oblique derivative boundary condition in the direction $\nu$ possibly depending on $x$ :

$$
\begin{cases}w_{t}+\mathcal{H}\left(t, x, D w, D^{2} w\right)=0 & (0, T) \times D \\ \langle\nu(x), D w\rangle=0 & (0, T) \times \partial D \\ w(0, x)=w_{0}(x) & \bar{D}\end{cases}
$$

where $D, \nu: \mathbb{R}^{n} \rightarrow \mathbb{R}^{n}, \mathcal{H}:[0, T] \times \mathbb{R}^{n} \times \mathbb{R}^{n} \times S_{n}\left(\mathcal{S}_{n}\right.$ is the space of $n \times n$ symmetric matrices), and $w_{0}: \mathbb{R}^{n} \rightarrow \mathbb{R}$ satisfy the following properties:

(P1) $\bar{D}$ is a locally compact subset of $\mathbb{R}^{n}$ such that there exists $b>0$ such that

$$
\begin{aligned}
& \bigcup_{0 \leq t \leq b} B_{t b}(x+t \nu(x)) \subset D^{c}, \quad \forall x \in \partial D \\
& \bigcup_{0 \leq t \leq b} B_{t b}(x-t \nu(x)) \subset \bar{D}, \quad \forall x \in \partial D
\end{aligned}
$$

(P2) $\mathcal{H} \in C\left(\mathbb{R} \times \bar{D} \times \mathbb{R}^{n} \times S_{n}\right)$ and there is a neighborhood $U$ of $\partial D$ in $\bar{D}$ and a function $\omega_{1} \in C([0, \infty])$ satisfying $\omega_{1}(0)=0$ such that $\forall t \in[0, T), x \in U, p, q \in \mathbb{R}^{n}, X, Y \in \mathcal{S}_{d}$

$$
|\mathcal{H}(t, x, p, X)-\mathcal{H}(t, x, q, Y)| \leq \omega_{1}(|p-q|+\|X-Y\|) ;
$$

(P3) There is a function $\omega_{2} \in C([0, \infty])$ satisfying $\omega_{2}(0)=0$ such that

$$
\begin{gathered}
\mathcal{H}(t, y, p,-Y)-\mathcal{H}(t, x, p, X) \leq \omega_{2}\left(\alpha|x-y|^{2}+|x-y|(|p|+1)\right) \\
\forall \alpha \geq 1, t \in[0, T), x, y \in \bar{D}, p \in \mathbb{R}^{n}, X, Y \in \mathcal{S}_{n} \text { such that } \\
-\alpha\left(\begin{array}{cc}
I & 0 \\
0 & I
\end{array}\right) \leq\left(\begin{array}{cc}
X & 0 \\
0 & Y
\end{array}\right) \leq \alpha\left(\begin{array}{cc}
I & -I \\
-I & I
\end{array}\right) .
\end{gathered}
$$

(P4) $\nu$ is $C^{2}\left(\mathbb{R}^{n}\right) \cap W^{2, \infty}\left(\mathbb{R}^{n}\right)$ with $|\nu(x)|=1$, and $w_{0}$ is a Lipschitiz continuous function.

It is clear that under assumption (H1)-(H2), equation (3.5) is a particular case of (A.1), with $n=d+1, \bar{D} \equiv \operatorname{Epigraph}(g), \nu \equiv-e_{y}, \mathcal{H}(t, \cdot) \equiv$ $H(T-t, \cdot)$. We notice also that under $(\mathrm{H} 1)-(\mathrm{H} 2)$, the set defined by $\bar{D}=$ $\operatorname{Epigraph}(g)$ satisfies the property $(\mathrm{P} 1)$, with $b:=1 / \sqrt{1+L_{g}^{2}}$. The properties (P2)-(P4) are also satisfied under (H1)-(H2).

In the sequel, it will be usefull to use the concept of semijets:

Definition A.1 (Parabolic semijets). Let $\mathcal{O}$ be a locally compact subset of $\mathbb{R}^{n}$. We define the parabolic semijets of a function $w:[0, T] \times \mathcal{O} \rightarrow \mathbb{R}$ in 
$(t, x) \in(0, T) \times \mathcal{O}$ by:

$$
\begin{aligned}
& \mathcal{P}^{1,2,+} w(t, x):=\left\{(a, p, X) \in \mathbb{R} \times \mathbb{R}^{n} \times \mathcal{S}_{n} \mid \text { as }(0, T) \times \mathcal{O} \ni(s, y) \rightarrow(t, x)\right. \\
& w(s, y) \leq w(t, x)+a(s-t)+\langle p, y-x\rangle+\frac{1}{2}\langle X(y-x),(y-x)\rangle \\
& \left.+o\left(|s-t|+|y-x|^{2}\right)\right\} \\
& \mathcal{P}^{1,2,-} w(t, x):=\left\{(a, p, X) \in \mathbb{R} \times \mathbb{R}^{n} \times \mathcal{S}_{n} \mid \text { as }(0, T) \times \mathcal{O} \ni(s, y) \rightarrow(t, x)\right. \\
& w(s, y) \geq w(t, x)+a(s-t)+\langle p, y-x\rangle+\frac{1}{2}\langle X(y-x),(y-x)\rangle \\
& \left.+o\left(|s-t|+|y-x|^{2}\right)\right\} .
\end{aligned}
$$

Then we define the closures of the previous sets:

$$
\begin{aligned}
\overline{\mathcal{P}}^{1,2,+} w(t, x):= & \left\{(a, p, X) \in \mathbb{R} \times \mathbb{R}^{n} \times \mathcal{S}_{n} \mid \exists\left(t_{k}, x_{k}, a_{k}, p_{k}, X_{k}\right)\right. \text { such that } \\
& \left(a_{k}, p_{k}, X_{k}\right) \in \mathcal{P}^{1,2,+} w\left(t_{k}, x_{k}\right) \text { and } \\
& \left.\left(t_{k}, x_{k}, w\left(t_{k}, x_{k}\right), p_{k}, X_{k}\right) \rightarrow(t, x, w(t, x), p, X)\right\} \\
\overline{\mathcal{P}}^{1,2,-} w(t, x):= & \left\{(a, p, X) \in \mathbb{R} \times \mathbb{R}^{n} \times \mathcal{S}_{n} \mid \exists\left(t_{k}, x_{k}, a_{k}, p_{k}, X_{k}\right)\right. \text { such that } \\
& \left(a_{k}, p_{k}, X_{k}\right) \in \mathcal{P}^{1,2,-} w\left(t_{k}, x_{k}\right) \text { and } \\
& \left.\left(t_{k}, x_{k}, w\left(t_{k}, x_{k}\right), p_{k}, X_{k}\right) \rightarrow(t, x, w(t, x), p, X)\right\}
\end{aligned}
$$

Theorem A.2. Assume (P1)-(P4) hold. Let u (resp. v) be a usc subsolution (resp. lsc super-solution) of (A.1) satisfying the following growth conditions

$$
\begin{aligned}
u(t, x) & \leq C(1+|x|), \\
\text { (resp. } \quad v(t, x) & \geq-C(1+|x|)) .
\end{aligned}
$$

Then $u \leq v$ on $[0, T] \times \bar{D}$.

Before starting the proof we state some important preliminary results.

Lemma A.3. If (P1) and (P4) hold, then there exists a family $\left\{w_{\varepsilon}\right\}_{\varepsilon>0}$ of $C^{2}$ functions on $\mathbb{R}^{n} \times \mathbb{R}^{n}$ and positive constants $\theta, C$ such that

$$
\begin{aligned}
\vartheta_{\varepsilon}(x, x) & \leq \varepsilon \\
\vartheta_{\varepsilon}(x, y) & \geq \theta \frac{|x-y|^{2}}{\varepsilon} \\
\left\langle\nu(x), D_{x} \vartheta_{\varepsilon}(x, y)\right\rangle & \geq-C \frac{|x-y|^{2}}{\varepsilon} \quad \text { if } y-x \notin \Gamma \\
\left\langle\nu(x), D_{y} \vartheta_{\varepsilon}(x, y)\right\rangle & \geq 0 \quad \text { if } x-y \notin \Gamma \\
\left|D_{y} \vartheta_{\varepsilon}(x, y)\right| & \leq C \frac{|x-y|}{\varepsilon}, \\
\left|D_{x} \vartheta_{\varepsilon}(x, y)+D_{y} \vartheta_{\varepsilon}(x, y)\right| & \leq C \frac{|x-y|^{2}}{\varepsilon}
\end{aligned}
$$

and

$$
D^{2} \vartheta_{\varepsilon}(x, y) \leq C\left\{\frac{1}{\varepsilon}\left(\begin{array}{cc}
I & -I \\
-I & I
\end{array}\right)+\frac{|x-y|^{2}}{\varepsilon} I_{2 d}\right\}
$$

for $\varepsilon>0$ and $x, y \in \mathbb{R}^{n}$.

Proof. The result is presented in [25]. 
Lemma A.4. If (P1) and (P4) hold, then there exists $h \in C^{2}(\bar{D})$ such that:

$$
h \geq 0 \quad \text { on } \bar{D} \quad \text { and } \quad h=0 \quad \text { in } \bar{D} \backslash U
$$

(where $U$ is a neighborhood of $\partial D$ as in Property (P2)) and

$$
\langle\nu(x), D h(x)\rangle \geq 1 \quad x \in \partial D .
$$

Proof. The result can be obtained adapting the arguments in [25], thanks to the local compactness of the set $D$.

Proof of Theorem A.2. We will prove the theorem for $u$ and $v$ sub- and super-solution of (A.1) with boundary condition respectively replaced by $\langle\nu(x), D u\rangle+\alpha$ and $\langle\nu(x), D v\rangle-\alpha$ for a certain $\alpha>0$. It means that on $(0, T) \times \partial D, u$ and $v$ satisfy

$$
\begin{aligned}
\min (\langle\nu(x), p\rangle+\alpha, a+\mathcal{H}(t, x, p, X)) \leq 0 & \forall(a, p, X) \in \overline{\mathcal{P}}^{1,2,+} u(t, x) \\
\max (\langle\nu(y), q\rangle-\alpha, b+\mathcal{H}(t, y, q, Y)) \geq 0 & \forall(b, q, Y) \in \overline{\mathcal{P}}^{1,2,-} v(t, y) .
\end{aligned}
$$

We observe that it is always possible to consider a sub-solution $u_{\gamma}$ such that

$$
\left\{\begin{array}{l}
\lim _{t \rightarrow T} u_{\gamma}(t, x)=-\infty \\
\partial_{t}\left(u_{\gamma}\right)+\mathcal{H}\left(t, x, D u_{\gamma}, D^{2} u_{\gamma}\right)<0
\end{array}\right.
$$

defining, for instance, $u_{\gamma}(t, x):=u(t, x)-\frac{\gamma}{T-t}$. The desired comparison result is then obtained as a limit for $\gamma \rightarrow 0$.

Given $\delta>0$, let us define $\rho_{\delta}:=\sup _{(t, x) \in[0, T) \times \bar{D}}\left(u(t, x)-v(t, x)-2 \delta\left(1+|x|^{2}\right)\right)$.

The growth condition on $u$ and $v$ implies that there exists $(s, z) \in[0, T) \times \bar{D}$ such that

$$
\rho_{\delta}=u(s, z)-v(s, z)-2 \delta\left(1+|z|^{2}\right) .
$$

Set $\rho:=\varlimsup_{\delta \rightarrow 0} \rho_{\delta}$. Assume $\rho \leq 0$. Since $u(t, x)-v(t, x) \leq 2 \delta\left(1+|x|^{2}\right)+\rho_{\delta}$, for every $(t, x) \in[0, T) \times \bar{D}$, we have that $u(t, x) \leq v(t, x)$ on $[0, T) \times \bar{D}$.

Now, assume that $\rho>0$ and let us show a contradiction. Thanks to (P1) and (P4), if $z \in \partial D$, then there exists $\delta \in\left(0, \frac{1}{2}\right)$ such that $B_{t \delta}(x+t \nu(z)) \subset D^{c}$ for $x \in B_{\delta}(z) \cap \partial D, t \in(0,2 \delta]$. Set $\Gamma:=\bigcup_{t>0} B_{t \delta}(t \nu(z))^{\circ}$. It comes that

$$
y-x \notin \Gamma, \quad \text { if } x \in \partial D \cap B_{\delta}(z)^{\circ}, y \in \bar{D} \cap B_{\delta}(z)^{\circ} .
$$

In what follows we will restrict our attention to the events on the set $B_{\delta}(z) \cap$ $\bar{D}$, then we can assume $\nu \in W^{2, \infty}\left(\mathbb{R}^{n}, \mathbb{R}^{n}\right)$ and $|\nu(x)|=1$.

Thanks to Lemma A.3 we can define

$\Phi(t, x, y):=u(t, x)-v(t, y)-\alpha|x-z|^{2}-\vartheta_{\varepsilon}(x, y)-\delta\left(1+|x|^{2}\right)-\delta\left(1+|y|^{2}\right)$. and thanks to the growth conditions and the semicontinuity of $u$ and $v$ we can state that there exists $(\bar{t}, \bar{x}, \bar{y}) \in[0, T) \times \bar{D}$ maximum point for $\Phi$. Thanks to A.2 and (A.3) the following inequalities hold

$$
\begin{aligned}
\rho_{\delta}-\varepsilon & \leq \Phi(s, z, z) \leq \Phi(\bar{t}, \bar{x}, \bar{y}) \\
& \leq u(\bar{t}, \bar{x})-v(\bar{t}, \bar{y})-\alpha|\bar{x}-z|^{2}-\theta \frac{|\bar{x}-\bar{y}|^{2}}{\varepsilon}-\delta\left(1+|\bar{x}|^{2}\right)-\delta\left(1+|\bar{y}|^{2}\right)
\end{aligned}
$$


and by classical arguments (see [21]), extracting a subsequence if necessary, we can conclude that

$$
\begin{gathered}
\frac{|\bar{x}-\bar{y}|^{2}}{\varepsilon} \stackrel{\varepsilon \rightarrow 0}{\longrightarrow} 0 \\
\bar{x}, \bar{y} \stackrel{\varepsilon \rightarrow 0}{\longrightarrow} z .
\end{gathered}
$$

If $\bar{t}=0$ :

$\rho_{\delta}-\varepsilon \leq \Phi(0, \bar{x}, \bar{y}) \leq w_{0}(\bar{x})-w_{0}(\bar{y})-\alpha|\bar{x}-z|^{2}-\theta \frac{|\bar{x}-\bar{y}|^{2}}{\varepsilon}-\delta\left(1+|\bar{x}|^{2}\right)-\delta\left(1+|\bar{y}|^{2}\right)$

so since the right-hand term in the inequality tends to zero for $\varepsilon, \delta \rightarrow 0$ and $\varlimsup_{\delta, \varepsilon \rightarrow 0}\left(\rho_{\delta}-\varepsilon\right)>0$, one can assume that $\bar{t}>0$ for $\varepsilon$ and $\delta$ small enough.

Let us define

$$
\begin{aligned}
& \tilde{u}(t, x):=u(t, x)-\alpha|x-z|^{2}-\delta\left(1+|x|^{2}\right) \\
& \tilde{v}(t, x):=v(t, x)+\delta\left(1+|x|^{2}\right) .
\end{aligned}
$$

Since $(\bar{t}, \bar{x}, \bar{y})$ is a maximum point for $\Phi$ and property A.8) holds, for $x$ and $y$ sufficiently close to $\bar{x}$ and $\bar{y}$, we have the following inequalities

$$
\begin{aligned}
& \tilde{u}(t, x)-\tilde{v}(t, y) \\
& \leq \tilde{u}(\bar{t}, \bar{x})-\tilde{v}(\bar{t}, \bar{y})+\vartheta_{\varepsilon}(x, y)-\vartheta_{\varepsilon}(\bar{x}, \bar{y}) \\
& \leq \tilde{u}(\bar{t}, \bar{x})-\tilde{v}(\bar{t}, \bar{y})+p \cdot(x-\bar{x})+q \cdot(y-\bar{y})+ \\
& +\frac{1}{2} D^{2} \vartheta_{\varepsilon}(\bar{x}, \bar{y})\left(\begin{array}{c}
x-\bar{x} \\
y-\bar{y}
\end{array}\right),\left(\begin{array}{c}
x-\bar{x} \\
y-\bar{y}
\end{array}\right)+O\left(|x-\bar{x}|^{3}+|y-\bar{y}|^{3}\right) \\
& \leq \tilde{u}(\bar{t}, \bar{x})-\tilde{v}(\bar{t}, \bar{y})+p \cdot(x-\bar{x})+q \cdot(y-\bar{y}) \\
& +\frac{C}{2}\left\{\frac{1}{\varepsilon}\left\langle\left(\begin{array}{cc}
I & -I \\
-I & I
\end{array}\right)\left(\begin{array}{l}
x-\bar{x} \\
y-\bar{y}
\end{array}\right),\left(\begin{array}{l}
x-\bar{x} \\
y-\bar{y}
\end{array}\right)\right\rangle\right. \\
& \left.+\left\langle\left(\begin{array}{cc}
I & 0 \\
0 & I
\end{array}\right)\left(\begin{array}{l}
x-\bar{x} \\
y-\bar{y}
\end{array}\right),\left(\begin{array}{l}
x-\bar{x} \\
y-\bar{y}
\end{array}\right)\right\rangle\right\}+O\left(|x-\bar{x}|^{3}+|y-\bar{y}|^{3}\right) \\
& \leq \tilde{u}(\bar{t}, \bar{x})-\tilde{v}(\bar{t}, \bar{y})+p \cdot(x-\bar{x})+q \cdot(y-\bar{y})+ \\
& +\frac{C}{2}\left\{\frac{1}{\varepsilon}|(x-\bar{x})-(y-\bar{y})|^{2}+\lambda|x-\bar{x}|^{2}+\lambda|y-\bar{y}|^{2}\right\},
\end{aligned}
$$

with $p=D_{x} \vartheta_{\varepsilon}(\bar{x}, \bar{y}), q=D_{y} \vartheta_{\varepsilon}(\bar{x}, \bar{y})$ and $\lambda=\frac{|\bar{x}-\bar{y}|^{2}}{\varepsilon}+\varepsilon$.

As a consequence of the Crandall-Ishii Lemma it follows that there exist $\tilde{X}, \tilde{Y} \in S_{m}$ and $a, b \in \mathbb{R}$ such that

$$
\begin{gathered}
-\frac{C}{\varepsilon} I_{2 m} \leq\left(\begin{array}{cc}
\tilde{X}-C \lambda I & 0 \\
0 & \tilde{Y}-C \lambda I
\end{array}\right) \leq \frac{C}{\varepsilon}\left(\begin{array}{cc}
I & -I \\
-I & I
\end{array}\right) \\
a+b=0
\end{gathered}
$$

and

$\left(a, D_{x} \vartheta_{\varepsilon}(\bar{x}, \bar{y}), \tilde{X}\right) \in \overline{\mathcal{P}}^{1,2,+} \tilde{u}(\bar{t}, \bar{x}) \quad\left(-b,-D_{y} \vartheta_{\varepsilon}(\bar{x}, \bar{y}),-\tilde{Y}\right) \in \overline{\mathcal{P}}^{1,2,-} \tilde{v}(\bar{t}, \bar{y})$. 
Recalling the definition of $\tilde{u}$ and $\tilde{v}$ one has

$$
\left(a, D_{x} \vartheta_{\varepsilon}(\bar{x}, \bar{y})+2 \alpha(\bar{x}-z)+2 \delta \bar{x}, \tilde{X}+2 \alpha I+2 \delta I\right) \in \overline{\mathcal{P}}^{1,2,+} u(\bar{t}, \bar{x})
$$

and

$$
\left(-b,-D_{y} \vartheta_{\varepsilon}(\bar{x}, \bar{y})-2 \delta \bar{y},-\tilde{Y}-2 \delta I\right) \in \overline{\mathcal{P}}^{1,2,-} v(\bar{t}, \bar{y}),
$$

so just setting $X:=\tilde{X}+2 \delta I$ and $Y:=Y+2 \delta I$ we get

$$
\left(a, D_{x} \vartheta_{\varepsilon}(\bar{x}, \bar{y})+2 \alpha(\bar{x}-z)+2 \delta \bar{x}, X+2 \alpha I\right) \in \overline{\mathcal{P}}^{1,2,+} u(\bar{t}, \bar{x})
$$

and

$$
\left(-b,-D_{y} \vartheta_{\varepsilon}(\bar{x}, \bar{y})-2 \delta \bar{y},-Y\right) \in \overline{\mathcal{P}}^{1,2,-} v(\bar{t}, \bar{y}),
$$

with

$$
-\frac{C}{\varepsilon} I_{2 m} \leq\left(\begin{array}{cc}
X-C \lambda I-2 \delta I & 0 \\
0 & Y-C \lambda I-2 \delta I
\end{array}\right) \leq \frac{C}{\varepsilon}\left(\begin{array}{cc}
I & -I \\
-I & I
\end{array}\right) .
$$

Let us assume that for $\varepsilon$ small enough

$$
\begin{array}{r}
\left\langle D_{x} \vartheta_{\varepsilon}(\bar{x}, \bar{y})+2 \alpha(x-z)+2 \delta \bar{x}, \nu(\bar{x})\right\rangle+\alpha>0 \\
\left\langle-D_{y} \vartheta_{\varepsilon}(\bar{x}, \bar{y})-2 \delta \bar{y}, \nu(\bar{y})\right\rangle-\alpha<0
\end{array}
$$

then by the definition of viscosity sub- and super-solution of A.1 we get

$$
a+\mathcal{H}\left(\bar{t}, \bar{x}, D_{x} \vartheta_{\varepsilon}(\bar{x}, \bar{y})+2 \alpha(\bar{x}-z)+2 \delta \bar{x}, X+2 \alpha I\right)<-c<0
$$

and

$$
-b+\mathcal{H}\left(\bar{t}, \bar{y},-D_{y} \vartheta_{\varepsilon}(\bar{x}, \bar{y})-2 \delta \bar{y},-Y\right) \geq 0
$$

and from (P2), (P3) A.14 and A.15), it follows

$$
\begin{aligned}
c<- & (a+b)+\mathcal{H}\left(\bar{t}, \bar{y},-D_{y} \vartheta_{\varepsilon}(\bar{x}, \bar{y})-2 \delta \bar{y},-Y\right) \\
& -\mathcal{H}\left(\bar{t}, \bar{x}, D_{x} \vartheta_{\varepsilon}(\bar{x}, \bar{y})+2 \alpha(\bar{x}-z)+2 \delta \bar{x}, X+2 \alpha I\right) \\
\leq & \mathcal{H}\left(\bar{t}, \bar{y},-D_{y} \vartheta_{\varepsilon},-Y+C \lambda I+2 \delta I\right)-\mathcal{H}\left(\bar{t}, \bar{x},-D_{y} \vartheta_{\varepsilon}, X-C \lambda I-2 \delta I\right) \\
& +\omega_{1}\left(\left|D_{x} \vartheta_{\varepsilon}+D_{y} \vartheta_{\varepsilon}\right|+2 \alpha+C \lambda+2 \delta(2+|\bar{x}|+|\bar{y}|)+2 \alpha|\bar{x}-z|\right) \\
\leq & \omega_{1}\left(\left|D_{x} \vartheta_{\varepsilon}+D_{y} \vartheta_{\varepsilon}\right|+2 \alpha+C \lambda+2 \delta(2+|\bar{x}|+|\bar{y}|)+2 \alpha|\bar{x}-z|\right)+ \\
& +\omega_{2}\left(C \frac{|\bar{x}-\bar{y}|^{2}}{\varepsilon}+|\bar{x}-\bar{y}|(2 \delta|\bar{y}|+1)\right)
\end{aligned}
$$

so that the desired contradiction is obtained by taking the limit for $\varepsilon, \alpha \rightarrow 0$. In order to prove (A.16) and A.17) we proceed as in [25]. In fact for $\varepsilon$ and $\delta$ sufficiently small

$$
\begin{aligned}
& \left\langle D_{x} \vartheta_{\varepsilon}(\bar{x}, \bar{y}), \nu(\bar{x})\right\rangle \geq-\frac{\alpha}{2} \Rightarrow\left\langle D_{x} \vartheta_{\varepsilon}(\bar{x}, \bar{y}), \nu(\bar{x})\right\rangle+\langle 2 \alpha(x-z), \nu(\bar{x})\rangle+2 \delta\langle\bar{x}, \nu(\bar{x})\rangle+\alpha>0 \\
& \left\langle D_{y} \vartheta_{\varepsilon}(\bar{x}, \bar{y}), \nu(\bar{y})\right\rangle \geq-\frac{\alpha}{2} \Rightarrow \quad-\left\langle D_{y} \vartheta_{\varepsilon}(\bar{x}, \bar{y}), \nu(\bar{y})\right\rangle-2 \delta\langle\bar{y}, \nu(\bar{y})\rangle-\alpha<0,
\end{aligned}
$$

then thanks to (A.4) and (A.5), A.16 and A.17) are finally obtained. In order to conclude the proof we need to argue how it is possible to modify our boundary conditions. 
Let us assume that $u$ and $v$ are respectively viscosity sub- and super-solution of the original problem. Thanks to Lemma A.4 we can define

$$
u_{\alpha}(t, x):=u(t, x)-\alpha h(x)-C t
$$

and

$$
v_{\alpha}(t, x):=v(t, x)+\alpha h(x)+C t .
$$

If $(a, p, X) \in \overline{\mathcal{P}}^{1,2,+} u_{\alpha}(t, x)$ and $(b, q, Y) \in \overline{\mathcal{P}}^{1,2,-} v_{\alpha}(t, x)$, by property (P2) one has

$$
\begin{aligned}
& a+\mathcal{H}(t, x, p, X) \leq a+C+\mathcal{H}\left(t, x, p+\alpha D h, X+\alpha D^{2} h\right)-C+\omega_{1}(\alpha M), \\
& b+\mathcal{H}(t, x, q, Y) \geq b-C+\mathcal{H}\left(t, x, q-\alpha D h, Y-\alpha D^{2} h\right)+C-\omega_{1}(\alpha M)
\end{aligned}
$$

and

$$
\begin{aligned}
\langle\nu(x), p\rangle & =\langle\nu(x), p+\alpha \operatorname{Dh}(x)\rangle-\alpha\langle\nu(x), \operatorname{Dh}(x)\rangle \\
& \leq\langle\nu(x), p+\alpha \operatorname{Dh}(x)\rangle-\alpha \\
\langle\nu(x), p\rangle & \geq\langle\nu(x), p-\alpha \operatorname{Dh}(x)\rangle+\alpha
\end{aligned}
$$

where $M:=\max \left(|D h|+\left\|D^{2} h\right\|\right)$ (from the construction of $h$ follows the boundness of $D h$ and $D^{2} h$ ).

Observing that

$$
\begin{aligned}
\overline{\mathcal{P}}^{1,2,+} u(t, x) & =\overline{\mathcal{P}}^{1,2,+} u_{\alpha}(t, x)+\left(C, \alpha D h, \alpha D^{2} h\right) \\
\overline{\mathcal{P}}^{1,2,-} v(t, x) & =\overline{\mathcal{P}}^{1,2,-} v_{\alpha}(t, x)-\left(C, \alpha D h, \alpha D^{2} h\right)
\end{aligned}
$$

one has

$$
\begin{aligned}
\left(a+C, p+\alpha D h(x), X+\alpha D^{2} h(x)\right) & \in \overline{\mathcal{P}}^{1,2,+} u(x) \\
\left(b-C, q-\alpha D h(x), Y-\alpha D^{2} h(x)\right) & \in \overline{\mathcal{P}}^{1,2,-} v(x)
\end{aligned}
$$

then, by the definition of viscosity sub- and super-solution

$$
\begin{aligned}
& \begin{cases}a+C+\mathcal{H}\left(t, x, p+\alpha D h, X+\alpha D^{2} h\right) \leq 0 & \text { on } D \\
\min \left(\langle\nu(x), p+\alpha D h(x)\rangle, a+C+\mathcal{H}\left(t, x, p+\alpha D h, X+\alpha D^{2} h\right)\right) \leq 0 & \text { on } \partial D\end{cases} \\
& \text { and } \\
& \begin{cases}b-C+\mathcal{H}\left(t, x, q-\alpha D h, Y-\alpha D^{2} h\right) \geq 0 & \text { on } D \\
\max \left(\langle\nu(x), p-\alpha D h(x)\rangle, b-C+\mathcal{H}\left(t, x, q-\alpha D h, Y-\alpha D^{2} h\right)\right) \geq 0 & \text { on } \partial D .\end{cases}
\end{aligned}
$$

For $\alpha$ small enough, taking $C=w_{1}(\alpha M)$, we can finally conclude by inequalities A.18, A.20 and A.19, A.21) that

$$
\begin{cases}a+\mathcal{H}(t, x, p, X) \leq 0 & \text { on } D \\ \min (\langle\nu(x), p\rangle+\alpha, a+\mathcal{H}(t, x, p, X)) \leq 0 & \text { on } \partial D\end{cases}
$$

and

$$
\begin{cases}+b+\mathcal{H}(t, x, q, Y) \geq 0 & \text { on } D \\ \max (\langle\nu(x), p\rangle-\alpha, b+\mathcal{H}(t, x, q, Y)) \geq 0 & \text { on } \partial D .\end{cases}
$$

In other words for $\alpha$ small enough $u_{\alpha}$ and $v_{\alpha}$ are respectively sub- and supersolution of A.1 with boundary conditions $\left\langle\nu(x), D u_{\alpha}\right\rangle+\alpha$ and $\left\langle\nu(x), D v_{\alpha}\right\rangle-$ 
$\alpha$ and since $u_{\alpha} \stackrel{\alpha \rightarrow 0}{\rightarrow} u$ and $v_{\alpha} \stackrel{\alpha \rightarrow 0}{\rightarrow} v$, we can prove the comparison theorem for them instead of $u$ and $v$.

\section{REFERENCES}

[1] Altarovici A., Bokanowski O., Zidani H. (2013) A general Hamilton-Jacobi framework for non-linear state-constrained control problems. ESAIM: COCV DOI: $10.1051 / \mathrm{cocv} / 2012011$.

[2] Aubin J.-P., Da Prato G. (1990) Stochastic viability and invariance. Ann. Scuola Norm. Sup. Pisa Cl. Sci. 595:613-17(4).

[3] Aubin J.-P., Da Prato G. (1998) The viability theorem for stochastic differential inclusions. Stochastic Anal. App. DOI:10.1080/07362999808809512.

[4] Barles G., Daher C., Romano M. (1994) Optimal control on the $L^{\infty}$ norm of a diffusion process. SIAM J. Control Optim. DOI: 10.1137/S0363012991223595.

[5] Barles G., Daher C., Romano M. (1995) Convergence of numerical schemes for parabolic equations arising in finance theory. Math. Models Methods Appl. Sci. DOI: 10.1017/CBO9781139173056.002.

[6] Barles G., Jakobsen E.R. (2002) On the convergence rate of approximation schemes for Hamilton-Jacobi-Bellman equations. M2AN Math. Model. Numer. Anal. DOI: 10.1051/m2an2:002002.

[7] Barles G., Jakobsen E.R. (2005) Error bounds for monotone approximation schemes for Hamilton-Jacobi-Bellman equations. SIAM J. Numer. Anal. DOI: 10.1137/S003614290343815X.

[8] BARles G., JAKOBSEn E.R. (2007) Error bounds for monotone approximation schemes for parabolic Hamilton-Jacobi-Bellman equations. Math. Comp. DOI: 10.1090/S0025-5718-07-02000-5.

[9] Barles G., Lions P.L. (1991) Fully nonlinear Neumann type boundary conditions for first-order Hamilton-Jacobi equations. Nonlinear Anal. Theory Methods Appl. DOI: 10.1016/0362-546X(91)90165-W.

[10] Barles G.,Souganidis P.E. (1991) Convergence of approximation schemes for fully nonlinear second order equations. Asymptotic Anal. DOI: 10.3233/ASY-1991-4305.

[11] BARron E.N. (1993) The Bellman equation for control of the running max of a diffusion and applications to lookback options. Appl. Anal. DOI: 10.1080/00036819308840158.

[12] Bokanowski O., Forcadel N., Zidani H. (2010) Reachability and minimal times for state constrained nonlinear problems without any controllability assumption. SIAM J. Control Optim. DOI: 10.1137/090762075.

[13] Bonnans J.F., Maroso S., Zidani H. (2006) Error estimates for stochastic differential games: the adverse stopping case. IMA, J. Numer. Anal. DOI: 10.1093/imanum/dri034.

[14] Bonnans J.F., Maroso S., Zidani H. (2007) Error bounds for a Stochastic Impulse Control Problem. Appl. Math Optim. DOI: 10.1007/s00245-006-0865-2.

[15] Bonnans J.F., Ottenwaelter E., Zidani H. (2004) Numerical schemes for the two dimensional second-order HJB equation. ENSAIM: M2AN 723:735-38.

[16] Bonnans J.F., ZIDANi H. (2003) Consistency of generalized finite difference schemes for the stochastic HJB equation. SIAM J. Numer. Anal. DOI: $10.1137 / \mathrm{S} 0036142901387336$.

[17] Bouchard B. (2002) Stochastic targets with mixed diffusion processes and viscosity solutions. Stochastic Processes and their Applications DOI: 10.1016/S03044149(02)00129-1.

[18] Bouchard B., Touzi N. (2011) Weak dynamic programming principle for viscosity solutions. SIAM J. Control Optim. DOI: 10.1137/090752328. 
[19] Camilli F., Falcone M. (1995) An approximation scheme for the optimal control of diffusion processes. RAIRO Modél. Math. Anal. Numér. 97:122-29(1).

[20] Capuzzo-Dolcetta I. (1983) On a discrete approximation of the Hamilton-Jacobi equation of dynamic programming. Appl. Math. Optim. DOI: 10.1007/BF01448394.

[21] Crandall M.G., Ishin H., Lions P.L. (1992) User's guide to viscosity solutions of second order partial differential equations. Bull. Amer. Math. Soc. DOI: 10.1090/S02730979-1992-00266-5.

[22] Crandall M.G., Lions P.L. (1984) Two approximations of solutions of HamiltonJacobi equations. Math. Comp. DOI:10.2307/2007396.

[23] Crandall M.G., Lions P.L. (1996) Convergent difference schemes for nonlinear parabolic equations and mean curvature motion. Numer. Math. DOI: $10.1007 / \mathrm{s} 002110050228$.

[24] Debrabant K., Jakobsen E.R. (2013) Semi-Lagrangian schemes for linear and fully non-linear diffusion equations. Math. Comp. DOI: 10.1090/S0025-5718-201202632-9.

[25] Dupuis P., Ishi H. (1990) On oblique derivative problems for fully nonlinear secondorder elliptic partial differential equations on nonsmooth domains. Nonlinear Anal. Theory Methods Appl. DOI: 10.1016/0362-546X(90)90048-L.

[26] Heinricher A. C., Stockbridge R. H. (1991) Optimal control of the running max. SIAM J. Control Optim. DOI: 10.1137/0329052.

[27] Ishir H. (1989) A boundary value problem of the Dirichlet type for Hamilton-Jacobi equations. Ann. Scuola Norm. Sup. Pisa Cl. Sci. 105:135-16(1).

[28] Ishi H. (1991) Fully nonlinear oblique derivative problems for nonlinear Second-order elliptic PDEs. Duke Math. J. DOI: 10.1215/S0012-7094-91-06228-9.

[29] Ishii H., Lions P.L. (1990) Viscosity solutions of fully nonlinear Second-order elliptic partial differential equations. J. Differential equations DOI: 10.1016/00220396(90)90068-Z.

[30] KRYLOV N.V. (1998) On the rate of convergence of finite-difference approximations for Bellman's equations. St. Petersburg Math. J. 639:650-9(3).

[31] KRYLOV N.V. (2000) On the rate of convergence of finite-difference approximations for Bellman's equations with variable coefficients. Probab. Theory Relat. Fields DOI: $10.1007 / \mathrm{s} 004400050264$.

[32] Kushner H.J. (1977)Probability Methods for Approximations in Stochastic Control and for Elliptic Equations. Academic Press, New York.

[33] Kushner H.J., Dupuis P. (2001) Numerical methods for stochastic control problems in continuous time. Springer-Verlag, New York.

[34] Lions P.L. (1985) Neumann type boundary conditions for Hamilton-Jacobi equations. Duke Math. J. DOI: 10.1215/S0012-7094-85-05242-1.

[35] Menaldi J.-L. (1989) Some estimates for finite difference approximations. SIAM J. Control Optim. DOI: 10.1137/0327031 .

[36] Osher S., Sethian A.J. (1988) Fronts propagating with curvature dependent speed: algorithms based on Hamilton-Jacobi formulations. J. Comp. Phys. DOI: 10.1016/00219991(88)90002-2.

[37] Soner H.M., Touzi N. (2002) Stochastic target problems, dynamic programming and viscosity solutions. SIAM J. Control Optim. DOI: 10.1137/S0363012900378863.

[38] Wilmott P. (2000) Paul Wilmott on quantitative finance. John Wiley \& Sons Ltd (Revised Ed. of "Derivatives"), England.

[39] Yong J., Zhou X.Y. (1999) Stochastic controls: Hamiltonian systems and HJB equations. Stochastic Modeling and Applied Probability. Springer-Verlag, New York.

LABORATOIRE JACQUES-LOUIS LIONS, UNIVERSITÉ PARIS-DIDEROT (PARIS 7) UFR DE MATHÉMATIQUES - BÂT. SOPHIE GERMAIN 5 RUE THOMAS MANN 75205 PARIS CEDEX 13 (BOKA@MATH.JUSSIEU.FR). 
PROJET COMMANDS, INRIA SACLAY \& ENSTA PARISTECH, 828 BOULEVARD DES MARÉCHAUX, 91762 PALAISEAU CEDEX, (ATHENA.PICARELLI@INRIA.FR).

MATHEMATICS DEPARTMENT (UMA), ENSTA PARISTECH, 828 BOULEVARD DES MARÉCHAUX, 91762 PALAISEAU CEDEX (HASNAA.ZIDANI@ENSTA.FR). 